\title{
Stair Climbing Control for 4-DOF Tracked Vehicle Based on Internal Sensors
}

\author{
Daisuke Endo, ${ }^{1}$ Atsushi Watanabe, ${ }^{1}$ and Keiji Nagatani ${ }^{2}$ \\ ${ }^{1}$ Graduate School of Engineering, Tohoku University, Aramaki-aza Aoba 468-1, Aoba-ku, Sendai 980-0845, Japan \\ ${ }^{2}$ New Industry Creation Hatchery Center, Tohoku University, Aramaki-aza Aoba 6-6-10, Aoba-ku, Sendai 980-8579, Japan \\ Correspondence should be addressed to Daisuke Endo; endo@frl.mech.tohoku.ac.jp
}

Received 6 May 2017; Accepted 13 August 2017; Published 11 October 2017

Academic Editor: Gordon R. Pennock

Copyright (c) 2017 Daisuke Endo et al. This is an open access article distributed under the Creative Commons Attribution License, which permits unrestricted use, distribution, and reproduction in any medium, provided the original work is properly cited.

In search-and-rescue missions, multi-degrees-of-freedom (DOF) tracked robots that are equipped with subtracks are commonly used. These types of robots have superior locomotion performance on rough terrain. However, in teleoperated missions, the performance of tracked robots depends largely on the operators' ability to control every subtrack appropriately. Therefore, an autonomous traversal function can significantly help in the teleoperation of such robots. In this paper, we propose a planning and control method for 4-DOF tracked robots climbing up/down known stairs automatically based on internal sensors. Experimental results obtained using mockup stairs verify the effectiveness of the proposed method.

\section{Introduction}

In search-and-rescue missions, it is widely known that multidegrees-of-freedom (DOF) tracked robots that are equipped with subtracks are very useful. Quince [1] is a typical example of such a multi-DOF tracked robot. It explored buildings that were affected by the meltdown accident in the Fukushima Daiichi nuclear power plant [2]. Such a multi-DOF tracked robot has main tracks that constitute a skid-steered mobile base, and each one rotates independently. In addition, it is equipped with subtracks that connect to the main body with rotary joints at the front and rear of the main tracks. Further, it has high traversability on rough terrain in spite of its relatively simple mechanism.

For search-and-rescue missions, a tracked vehicle is sometimes required to traverse stairs to go to another floor and expand the reachable area. However, stairs represent typical uneven ground and are likely to cause some trouble for ground vehicles, even a multi-DOF tracked robot. In particular, if its subtracks are operated inadequately, it can cause fatal failure modes, such as tipping over. Moreover, in teleoperated missions, the operation of the subtracks is a difficult task, and this can easily cause operation errors. Therefore, it requires sophisticated operator skills.
To solve this problem, various related studies have been carried out on tracked robots [3-12]. Okada et al. realized a shared autonomous system to control subtracks adaptively by mounting laser range sensors on the multi-DOF tracked robot to detect the ground shape under the robot body [3] $\mathrm{Li}$ et al. developed an autonomous system to traverse stairs by using kinect sensor [5]. Zhang et al. realized climbing control including the detection of stairs, using a multi-DOF tracked robot equipped with a laser range finder, sonar, and a proximity distance sensor [9]. As mentioned in each paper, these are effective systems. However, each approach requires dedicated external sensors to detect the shape of the stairs. On the other hand, depending on the search target, there are cases where the shape of the stairs is known or where it can be detected using external sensors that were originally installed to achieve automatic navigation or environmental map construction. In such cases, it should be possible to reduce the number of dedicated external sensors that detect the shape of the road surface, which is required by the conventional method. As other related research examples, Ohno et al. proposed a semiautonomous approach to make the subtracks remain in contact with the unknown shape ground by realizing compliant joints that are based on jointtorque information [12]. However, this approach requires a 
current sensor on each subtrack to obtain the joint torque, and the sensor information is affected by the friction of its transmissions. To reduce the effect of the friction, the reduction ratio of the gear should be small. However, the sutrack power that is required to achieve control on uneven terrain is limited. In addition, Nan et al. proposed and verified control while ascending stairs by using a crawlertype robot without any additional sensors, but this method applies to the specific robot used in their study. Therefore, it is not considered suitable for general applications. The above conventional approaches are primarily for unknown natural rough terrain. On the other hand, there are some cases where the shape of the stairs is known, and in such simple cases, the robot does not require external sensors. Therefore, in this paper, we propose an autonomous motion for a 4DOF tracked robot for climbing up/down a known flight of stairs based on the rotational velocity of the main tracks, the rotational position of the subtracks, and IMU (Inertial Measurement Unit) information, without any other external and internal sensors. Besides, some conventional multi-DOF tracked robots, such as Quince, are 6-DOF and have four subtracks, one each at the front-left, front-right, rear-left, and rear-right of the main tracks. However, in the case when it is undergoing a typical climbing up/down motion on stairs, the left and right subtracks are not operated independently but are synchronized. Therefore, our proposal is also useful for 6DOF tracked robots. In addition, some verification tests are reported to confirm the validity of the proposed method.

As a supplement, we have already reported a part (only climbing up the stairs) related to this theme [13]. This article has comprehensive contents including climbing up and climbing down stairs in addition to [13].

\section{Problem Description}

In this study, we aim to realize a motion planning and control for a 4-DOF tracked robot to ascend and descend on various types of stairs by utilizing only the rotational information of each track and the attitude of the robot body. To proceed this study, we assumed the target robots and target environments, as shown in the following subsections. These assumptions are common for many applications such as autonomous inspection in known buildings or search-and-rescue, and therefore the results of this research can be used widely and generally.

2.1. Target Robot. In this study, we employ a 4-DOF tracked robot that consists of two main tracks to enable skid steering. It has two pairs of two synchronous subtracks. One pair is on both sides of the front, and the other is on both sides of the rear. The rotation range of each subtrack exceeds $\pm 90^{\circ}$ with respect to the front-rear direction of the main tracks. For joint angles, both the front and rear are defined as positive in case directed to upward toward main track. We also assume that the shape of the robot and the parameters of the position of the centroid of the robot are known. In addition, we assume that the position of the centroid of the robot is set such that it fits within the range that is directly above the contacting ground of the main track. Further, this position is in the state where the robot is grounded only by the main crawler onto the horizontal plane for any joint angle of the front and rear subtracks. In addition, there are multiple grousers on the periphery of each track that serve to improve the friction between the track and the ground. These should be sufficient to prevent the robot from slipping. We assume that the number of rotations of the main tracks and the subtracks are obtained by internal sensors, such as rotary encoders, which enable basic velocity/position control and odometry. The robot's pose is obtained by an IMU that contains acceleration sensors and gyroscopes. The definition of each specification parameter is described in Figure 1(i). $\Delta_{f}$ and $\Delta_{r}$ are the angles formed by the outer edges with respect to the symmetry line when the front and rear subtracks are viewed from the side of the robot, respectively. They are represented by the following:

$$
\begin{gathered}
\Delta_{f}=\arctan \left(\frac{r_{f}-r_{m}}{l_{f}}\right) \\
\Delta_{r}=\arctan \left(\frac{r_{r}-r_{m}}{l_{r}}\right) .
\end{gathered}
$$

2.2. Target Environment. In this paper, we deal with an environment consisting of two different horizontal floors that are connected by stairs, with all steps in parallel. The distance (pitch) $p$ and inclination $\theta_{s}$ between two adjacent edges of the stairs are constant.

We assume that all steps have a horizontal plane, and the existence of a vertical plane is arbitrary. $p, \theta_{s}$, and the number of steps $n$ are known. The width of the stairs is sufficiently larger than that of the robot. In addition, we assume that the relationship between $p$ and the maximum length of the flat area under the robot $L=l_{f}+l_{m}+l_{r}$ is $L \geq 2 p$. This assumption means that the robot can traverse the stairs while maintaining an inclination of the robot that is equal to that of the stairs. In addition, the conditions of $\left(p, \theta_{s}\right)$ are limited in that falling backward (described later) does not occur in the case where the robot stretches all subtracks completely straight. This assumption physically means that the robot is at least able to traverse the stairs.

\section{Definitions of Motion Phases and Failure Modes}

When the tracked robot climbs up the stairs, there are three motion phases (Figure 2). On the other hand, there are four motion phases in the case involving climbing down the stairs (Figure 3). In both cases (climbing up and climbing down), the failure mode that most easily occurs differs based on the motion phase. In this section, we define failure modes, motion phases, and their relationships.

3.1. Definition of the Three Phases of the Climbing-Up Motion. First, we divide the stair climbing-up motion into three phases as follows:

(i) Pitch-up ascend phase: the phase starts when the robot touches the lowest step of the stairs and lasts 


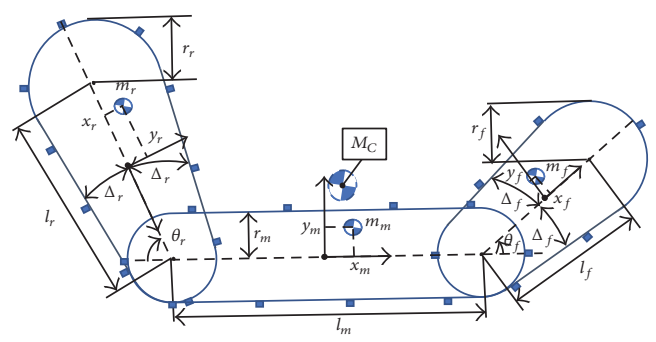

$m_{f}:$ mass of front subtracks
$m_{m}:$ mass of main tracks
$m_{r}:$ mass of rear subtracks
$M_{C}:$ centroid of the robot
$\theta_{f}:$ joint angle of front subtracks
$\theta_{r}:$ joint angle of rear subtracks
$l_{f}:$ length of front sub tracks
$l_{m}:$ length of main tracks

$l_{r}$ : length of rear subtracks $r_{f}$ : tip track radius of front subtracks $r_{m}:$ track radius of main tracks $r_{r}$ : tip track radius of rear subtracks $x_{f}, y_{f}: x$ - and $y$-coordinate of front subtrack's centroid $x_{m}, y_{m}: x$-and $y$-coordinate of main track's centroid $x_{r}, y_{r}: x$-and $y$-coordinate of rear subtrack's centroid

(i)

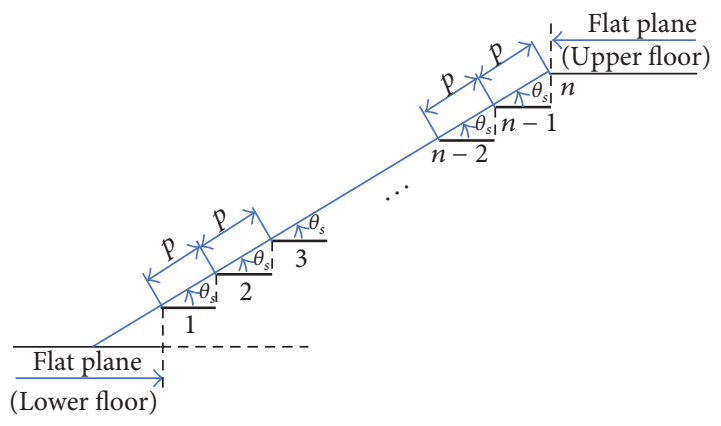

(ii)

FIGURE 1: Assumed shape of the robot and the stairs.

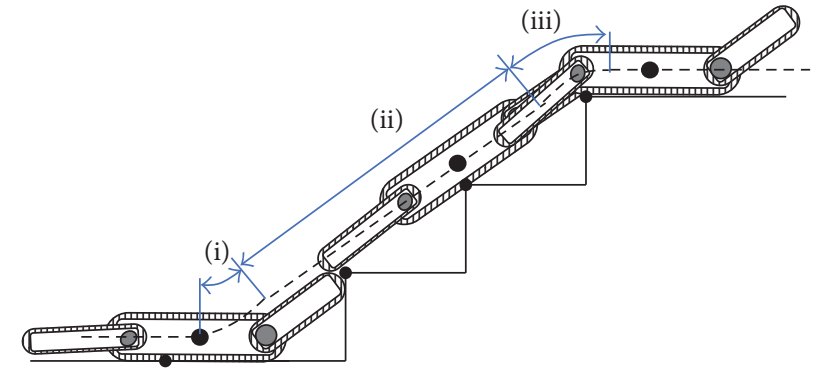

- - Trajectory of a representative position of the robot

FIgURE 2: Three motion phases when climbing up.

until the inclination of the robot, $\theta_{p}$, corresponds to that of the stairs, $\theta_{s}$ (Figure 2(i)).

(ii) Normal climbing-up phase: this is the phase between (i) and (iii). During this phase, the inclination angle of the robot $\theta_{p}$ is equal to that of the stairs $\theta_{s}$ (Figure 2(ii)).

(iii) Pitch-down ascend phase: this is the phase during which the inclination angle of the robot starts to

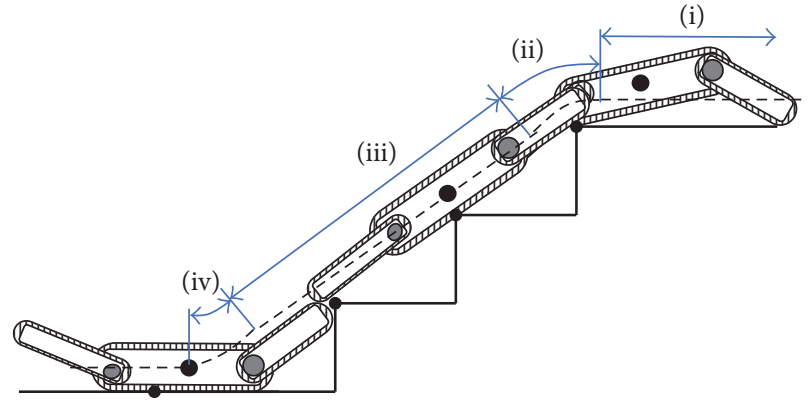

- - - Trajectory of a representative position of the robot

FIGURE 3: Four motion phases when climbing down.

decrease, which leads to the robot successfully reaching the new level (Figure 2(iii)).

3.2. Definition of the Four Phases of the Climbing-Down Motion. Secondly, we divide the stair climbing down motion into four phases as follows:

(i) Descend-ready phase: an operation state for detecting the relative position between the robot and the highest step. 


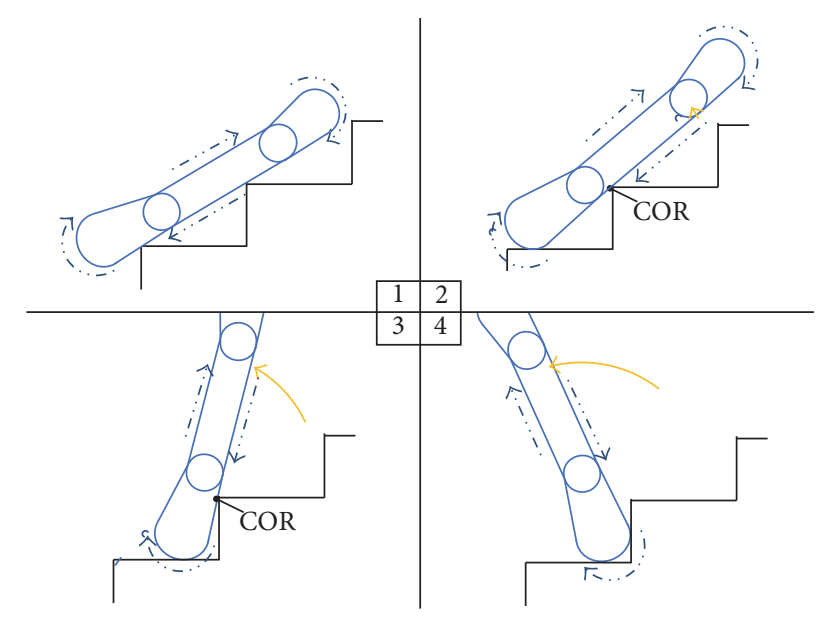

- .> Moving direction of track's periphery

$\longrightarrow$ Moving direction of robot's body

FIgURE 4: Falling backward.

(ii) Pitch-up descend phase: after the main tracks touch the highest step, the operation state until the pitch angle of the robot body $\theta_{p}$ substantially matches the inclination of the stairs $\theta_{s}$.

(iii) Normal climbing-down phase: this is the phase between (ii) and (iv). During this phase, the inclination angle of the robot, $\theta_{p}$, is equal to that of the stairs, $\theta_{s}$ (Figure 3(iii)).

(iv) Pitch-down descend phase: the phase from leaving the lowest step until the pitch angle of the robot body, $\theta_{p}$, evenly matches the lower floor

3.3. Definition of Failure Modes. While the robot traverses the stairs, it is necessary to prevent the following four failure modes:

(1) Slipping: the friction between the tracks and the steps is not sufficient to move in the direction of the main tracks.

(2) Falling backward: the robot body rotates backward and downward around the lowermost point of contact with the stairs (flips over backwards, as shown in Figure 4).

(3) Direction error: orientation of the robot deviates from the perpendicular direction to each edge of the stairs. Although this deviation is not a failure, as a result of this, falling sideward (tips sideways, as shown in Figure 5) or contact with side wall restricting the width of the stairs or slipping sideward (the frictional force decreases and slides down in the side direction of the robot) can happen. In this paper, as a generic name of these failure modes, it is written as direction error.
(4) Excessive shock: the contact shock exceeds the acceptable level for the robot (or surrounding environment) when it falls and touches the surrounding environment (as shown in Figure 6).

\section{Relationships between Motion Phases and Failure Modes}

The tendency of each failure mode is different for each motion phase described above. In this section, we explain the relationships with each failure mode that is likely to occur in each motion phase.

\subsection{Case Involving Climbing Up}

4.1.1. Pitch-Up Ascend Phase. Slipping tends to occur owing to an insufficient frictional force. On the other hand, because the pitch of the robot body is zero or is sufficiently small, it is difficult for it to fall backward and sideward compared to other motion phases. In addition, with respect to the excessive shock, it occurs if the robot body slips after being lifted in the pitch direction. However, this can be prevented by preventing it from slipping. Therefore, for this phase, it is desirable to prioritize the prevention of sliding.

4.1.2. Normal Climbing-Up Phase. According to the assumption described in Section 2, friction is sufficiently generated with respect to the staircase during the normal climbingup phase. For this reason, it is difficult to slip. On the other hand, because the inclination of the robot body is large, falling backward and sideward are likely to occur. In addition, excessive shock is unlikely to occur, with the exception of those caused by falling backward and sideward. In addition, even if it occurs, its influence tends to be reduced. Therefore, in this phase, it is preferable to prevent falling backward and sideward.

4.1.3. Pitch-Down Ascend Phase. As well as the normal climbing-up phase, slipping is unlikely. In addition, once falling backward and sideward can be prevented in the normal climbing-up phases, it is difficult for slippage to occur, even in this phase. On the other hand, if the subtracks cannot be properly operated, immediately after the centroid of the robot body passes through the highest edge of the step, it will be pulled by gravity and will fall while rotating around the edge, causing excessive shock when landing on the upper floor. Therefore, in this phase, it is desirable to prioritize the prevention of excessive shocks.

\subsection{Case Involving Climbing Down}

4.2.1. Descend-Ready Phase. Because this phase is primarily an operation to move on the upper even plane, it is difficult for any failure mode described in Section 3.2. However, depending on the position of the centroid of the robot and the length of the subtracks, it can fall over when the subtracks are grounded and the robot body is lifted. 


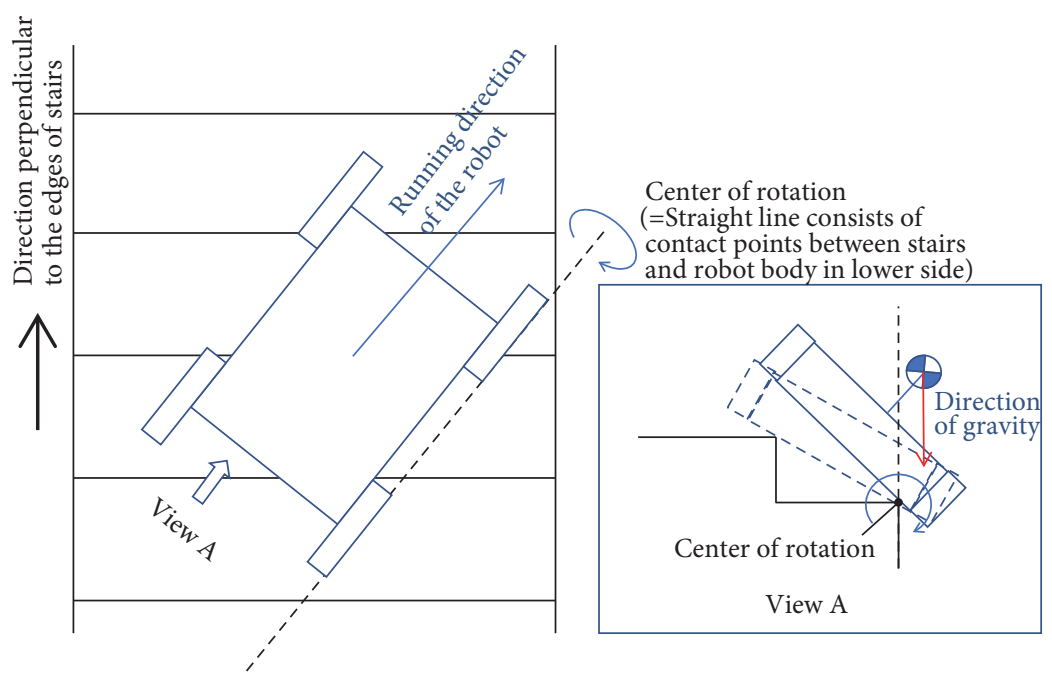

FIGURE 5: Falling sideward caused by direction error.

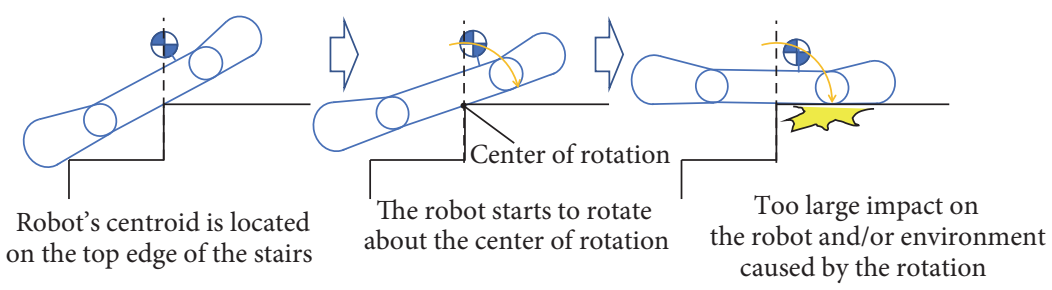

FIGURE 6: Excessive shock.

4.2.2. Pitch-Up Descend Phase. Because the grouser of the crawler belt comes into contact with the highest edge of the step in an oblique direction during this phase, friction is sufficiently generated (described later). Therefore, it is difficult for slippages to occur. In addition, because the pitch of the robot body is zero or sufficiently small, it is difficult to fall backward and sideward compared to the normal climbing-down phase described later. On the other hand, if it fails to properly operate the subtracks, it is pulled by gravity immediately after the centroid of the robot passes the edge of the highest step, falling while rotating, and an excessive shock occurs at the time of landing. Therefore, in this phase, it is also desirable to prioritize the prevention of excessive shocks.

4.2.3. Normal Climbing-Down Phase. This is the same as the normal climbing-up phase. Therefore, in this phase, it is preferable to prevent falling backward and sideward.

4.2.4. Pitch-Down Descend Phase. In addition to the pitch-up ascend phase, slipping is likely to occur. However, because the destination after slipping down is the lower floor on which the robot is going to advance, the sliding itself is not fatal. However, it is possible that the shock at the time of landing as a result of slipping may be excessive. On the other hand, it is difficult for falling backward and sideward to occur in this phase as long as they can be prevented during the normal climbing-down phase grade. Moreover, if the front subtracks cannot be properly operated, it falls from the lowest contacting edge immediately after the front subtracks leave, and an excessive shock is generated at the time of landing. Therefore, in this phase, it is desirable to give priority to preventing any excessive shocks, including those caused by sliding (Figure 7).

The relationship between each motion phase and failure mode described in this section is summarized in Table 2.

\section{Operation Strategy}

For the three phases involving climbing up the stair and the four phases involving climbing down, the failure modes that are likely to occur and which should be considered are different in each phase. In this section, we describe the operation strategy to prevent failure modes that are likely to occur in each phase. In addition, when the robot straightens the subtracks and the contact plane composed of the main tracks and the subtracks is in a horizontal state, the position of the entire centroid of the robot is generally not at the center of the contact plane and is either at the front or rear. In this paper, the side at which the centroid position is biased is defined as the front direction of the robot. Considering the situation with normal climbing up or down, it is desirable that the centroid be biased toward the upper side in order for the robot to operate without falling backward. This is common in ascending and descending cases. Therefore, it is desirable that the ascending operation be performed by advancing (translational speed is positive), and 


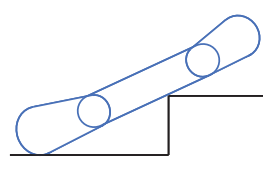

The robot starts to contact lower floor on rear subtracks

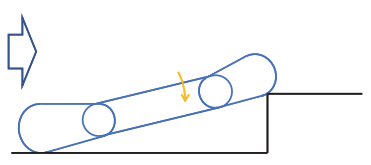

The robot's inclination reduces slowly Large impact happens when while lowest edge of stairs contacts lowest edge of stairs separates the robot

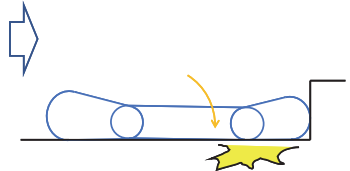

from the robot

FIgURE 7: Excessive shock can be generated in pitch-down descend phase.

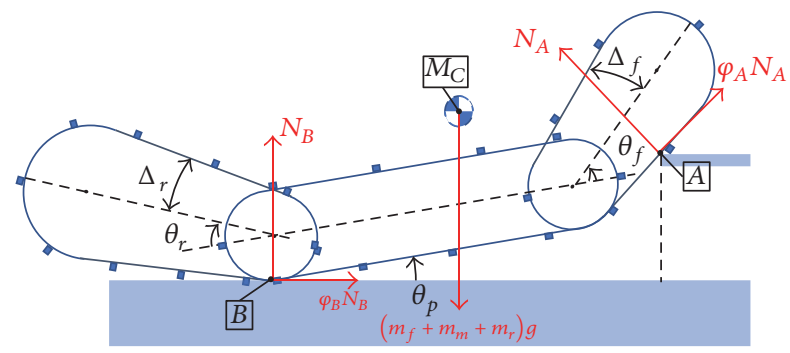

$A$ : forward contact point

$B$ : backward contact point

$N_{A}$ : normal reaction affecting contact point $A$
$N_{B}$ : normal reaction affecting contact point $B$ $\varphi_{A}:$ coefficient of friction at contact point $A$ $\varphi_{B}$ : coefficient of friction at contact point $B$

FIGURE 8: Physical model in the pitch-up phase.

the descending operation by reversing (translational speed is negative). In this paper, we assume that the climbing up is a forward movement and that the climbing down is a backward movement. Therefore, note that the side closer to the lower floor is the rear of the robot, and the side closer to the upper floor is the front, without depending on the difference between the climbing up and down movements.

5.1. Slip Prevention in Pitch-Up Ascend Phase. In the pitchup ascend phase, the operation to prevent slipping should be given priority. Thus, the motion of the robot should be predetermined in advance to prevent the slipping mode. For this reason, it is desirable that the reference joint angles of the subtracks $\theta_{f}$ and $\theta_{r}$ be set such that they increase friction. According to Y. Liu and G. Liu, when a tracked robot with grousers makes contact at the peak of a step, as shown in Figure 8, the equivalent friction coefficient (the value calculated from the force affecting it in the peripheral direction divided by the force affecting it in the normal direction) at the contact point $A \varphi_{A}$ can be calculated by [14]

$$
\varphi_{A}=\frac{\sin \left(\theta_{f}+\theta_{p}-\Delta_{f}\right)+\mu \cos \left(\theta_{f}+\theta_{p}-\Delta_{f}\right)}{\cos \left(\theta_{f}+\theta_{p}-\Delta_{f}\right)-\mu \sin \left(\theta_{f}+\theta_{p}-\Delta_{f}\right)},
$$

where $\mu$ is the friction coefficient between the materials of the grousers and the materials of the stairs. Therefore, the friction of the front subtrack is maximized when the denominator on the right side of (2) is equal to zero. Hence, the slipping mode can be minimized when the reference angle of the front subtrack $\theta_{f}^{\text {ref }}$ is determined as

$$
\theta_{f}^{\mathrm{ref}}=\arctan \left(\frac{1}{\mu}\right)-\theta_{p}+\Delta_{f} .
$$

Incidentally, for this condition, $\varphi_{A}$ mathematically equals infinity. This means that the finite force affects the contact point $A$ even if the normal force equals zero by the actual hooking of the grousers. In addition, in the case where the robot lifts up its main body from the horizontal state, the friction coefficient required to lift the body becomes smaller as the front contacting point becomes physically closer to the rear contacting point [15].

On the other hand, to prevent the falling backward mode, it is desirable that the rear subtracks remain very close to the ground to prevent it from falling backward. Based on the above, we can conclude that the reference joint angle of the rear subtrack $\theta_{r}^{\text {ref }}$ is determined as follows:

$$
\theta_{r}^{\mathrm{ref}}=\theta_{p}+\Delta_{r}+\delta
$$

where $\delta$ is the margin of the rear subtrack that does not make contact with the lower floor, even if there is any error or delay of control.

5.2. Prevention of Falling Backward and Sideward in Normal Climbing-Up Phase. In the normal climbing phase, two falling modes, falling backward and direction error, easily occur. It is possible to prevent the falling backward mode when the subtracks are operated properly. On the other hand, it is difficult to prevent the falling sideward mode in the 
case where the robot's orientation differs from the vertical direction of the edges of the stairs, even if the subtracks are operated properly. In the following subsections, we describe control methods to prevent the above two failure modes.

5.2.1. Prevention of Falling Backward Using Subtracks. The falling backward mode occurs in the case where the zeromoment point (namely, the acceleration projection point of the centroid to the support polygon) [16] of the robot moves downward and moves out of the range of the support polygon. Thus, when the robot climbs up the stairs, it is desirable that the centroid be located as low and frontward as possible and also extend the support polygon backward. Based on the above, it is effective for the front subtrack to be extended frontward, and the rear subtrack is extended backward so that all of the contact points are located in a single flat plane. Hence, the reference joint angles of the subtracks in the normal climbing-up phase are described by

$$
\begin{aligned}
& \theta_{f}^{\text {ref }}=\Delta_{f} \\
& \theta_{r}^{\text {ref }}=\Delta_{r} .
\end{aligned}
$$

5.2.2. Prevention of Direction Error Using Main Tracks. To prevent the robot from direction error, it is desirable that the orientation of the robot matches the vertical direction of the stairs as closely as possible. Therefore, the main tracks need to be controlled based on the control law of (6) using $\phi$, which is the angle between the orientation of the robot and the direction of the stairs:

$$
\Omega^{\mathrm{ref}}=-k_{\phi} \phi
$$

where $\Omega^{\text {ref }}$ is the target angular velocity of the robot body and $k_{\phi}$ is the control gain. The problem to be overcome involves the detection of $\phi$. In our assumption, the robot does not have any external sensors. Furthermore, in the case where the left and right main tracks experience unequal degrees of slipping on the stairs, and where the yaw angle of the robot obtained by a gyroscope experiences some drift, it causes an increase in the estimation error of $\phi$ when the robot relies only on a dead-reckoning method such as odometry or its gyroscope. Therefore, to solve this problem, we propose a method to calculate $\phi$ based on the roll information in the gravity direction. Let $\vec{y}$ be a unit vector pointing to the left of the robot body in the robot coordinate system, and let $\vec{g}$ be a unit vector in the gravity direction (Figure 9).

$$
\begin{aligned}
& \vec{y}=\left(-\cos \phi, \sin \phi \cos \theta_{s}, \sin \phi \sin \theta_{s}\right), \\
& \vec{g}=(0,0,-1),
\end{aligned}
$$

As $\vec{y} \cdot \vec{g}=\cos \left(\pi / 2+\theta_{R}\right)$, where $\theta_{R}$ is the roll angle of the robot body with respect to the gravity direction, we derive

$$
\phi=\arcsin \left(\frac{\sin \theta_{R}}{\sin \theta_{s}}\right) .
$$

Equation (8) indicates that we can obtain $\phi$ from the inclination of the stairs $\theta_{s}$ and the roll angle of the robot body

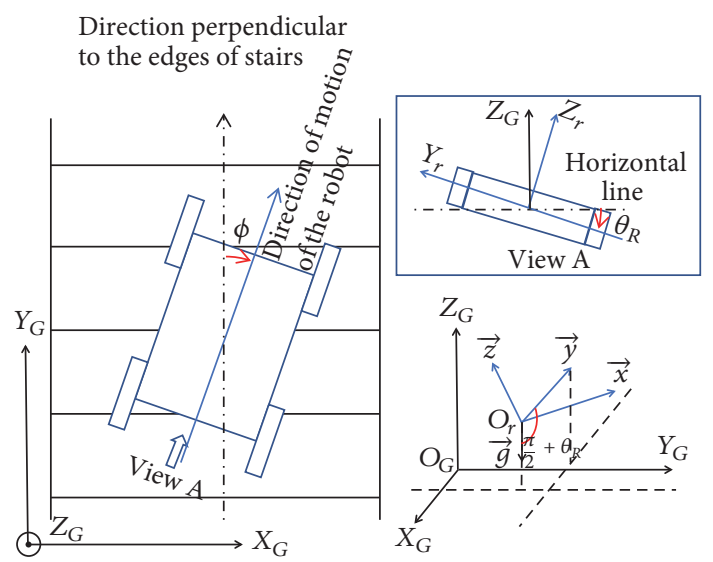

Figure 9: Vertical climbing direction and orientation of the robot.

$\theta_{R} . \theta_{s}$ is also known, and $\theta_{R}$ can be detected using only an internal sensor, IMU, without drift. Besides, the switch of control law from the pitch-up phase to the normal climbing phase is conducted when the pitch angle of the robot body $\theta_{p}$ comes sufficiently close to the inclination of the stairs, $\theta_{s}$. More specifically, the control law is changed when the condition

$$
\theta_{p} \geq \theta_{s}-e_{p}
$$

is established. Here, $\pm e_{p}$ describes the detection error range of the pitch angle of the robot.

5.3. Shock Mitigation in Pitch-Down Ascend Phase. In the pitch-down ascend phase, the robot should be very careful to mitigate the shock that is encountered between the robot body and the ground when it touches down on the upper floor. For shock mitigation, the ideal of a soft, landing motion makes the height of free fall equal to zero. This motion can be created theoretically by causing the front subtrack to make contact with the upper floor just before the centroid projection point of the robot body passes the peak of the final step. This can be realized only when the position of the robot is estimated without error. However, it is difficult for the robot to maintain the accuracy of the position estimation at high quality on stairs on which it tends to experience slipping or oscillation. In the case where the front subtracks contact the upper floor with the robot's position estimation error, it may hit the peak of the final step or the upper floor with some force. As a result, $\theta_{p}$ increases, and in the worst case, the falling backward mode occurs. To avoid the above situation, in this paper, we propose a method that absorbs the position estimation error for practical applications. Specifically, the robot operates in a motion such that its height of free fall does not exceed the maximum acceptable height $h_{\max }$ for the robot and the surrounding environment when the robot touches down on the upper floor.

Figure 10(a) indicates a state in which the joint angles of the rear subtracks are equal to zero, and the zero-moment point matches the peak of the final step. This is the statetransition configuration for which the robot starts to fall in a 


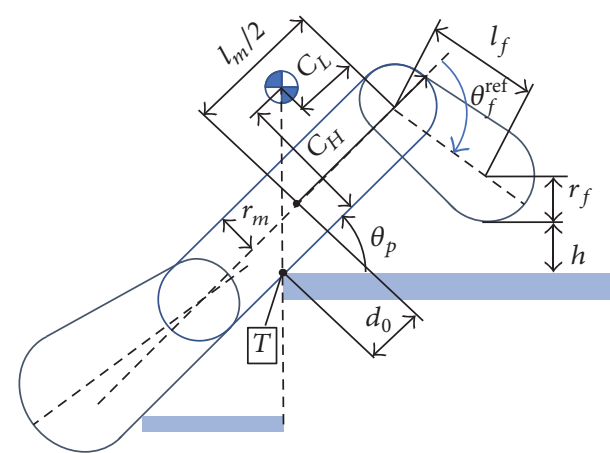

(a) $\theta_{f}^{\mathrm{ref}}$

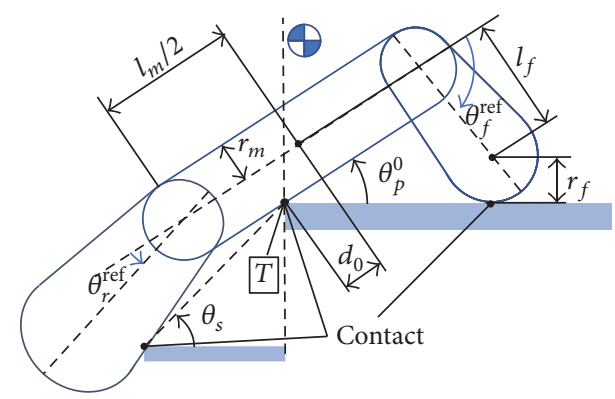

(b) $\theta_{r}^{\text {ref }}$

FIGURE 10: Target angle of subtracks in pitch-down mode.

forward direction, and $h$ indicates the height of free fall. Based on the geometric condition of Figure 10(a), the reference joint angle of the front subtrack should be $\theta_{f}^{\text {ref }}$, as described in (10) below, to make the height of free fall equal to the intended $h$ less than $h_{\max }$.

$$
\begin{aligned}
\theta_{f}^{\mathrm{ref}} & \\
= & \arcsin \left(\frac{\left(d_{0}+l_{m} / 2\right) \sin \theta_{s}+r_{m} \cos \theta_{s}-r_{f}-h}{l_{f}}\right) \\
& -\theta_{s},
\end{aligned}
$$

where $d_{0}$ is the offset distance in the front-back direction of the robot between the peak of the final step and the center of the main tracks, as shown in Figure 10(a). Specifically, it is expressed by the following equation:

$$
d_{0}=C_{H} \tan \theta_{p}+C_{L}-\frac{l_{m}}{2} .
$$

On the other hand, the joints of the rear subtracks should be controlled downward slightly, to support the robot body falling forward smoothly, as shown in Figure 10(b). We can determine a suitable angle of the rear subtrack by using the configuration of the robot in the figure: the three contact points do not generate the internal force required to grasp the stairs.

Depending on the geometric condition, the reference joint angle of the rear subtrack $\theta_{r}^{\text {ref }}$ can be calculated by

$$
\begin{aligned}
\theta_{r}^{\mathrm{ref}}= & -\arctan \left(\frac{p \sin \theta_{s}-\left(l_{m} / 2-d_{0}\right) \sin \theta_{p}^{0}}{p \cos \theta_{s}-\left(l_{m} / 2-d_{0}\right) \cos \theta_{p}^{0}}\right)+\theta_{p}^{0} \\
& +\Delta_{r},
\end{aligned}
$$

where $\theta_{p}^{0}$ is the pitch angle of the robot body when the front subtrack makes contact with the upper floor after the robot falls down in the forward direction. Thus, it satisfies

$$
\begin{aligned}
& \left(\frac{l_{m}}{2}+d_{0}\right) \sin \theta_{p}^{0}+r_{m} \cos \theta_{p}^{0}+l_{f} \sin \left(\theta_{f}+\theta_{p}^{0}\right)-r_{f} \\
& =0
\end{aligned}
$$

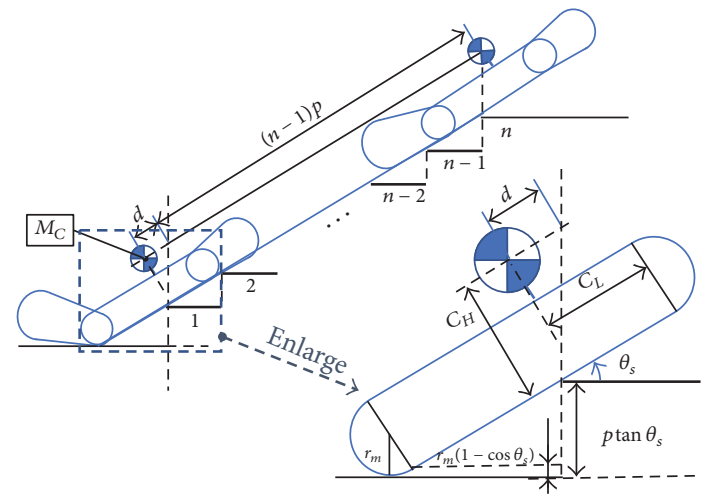

FIgURE 11: Distance to move in the normal climbing phase.

In this study, we assumed that the robot has no external sensors. Therefore, the phase transition from the normal climbing phase to the pitch-down phase is conducted when its running distance $D$ in the normal climbing phase exceeds a fixed value, as shown in the following equation:

$$
D \geq d+(n-1) p
$$

where $n$ is the number of steps. $d$ is a dimension that is indicated in Figure 11, which according to the geometric condition is determined by

$$
d=C_{L} \tan \theta_{s}+\frac{p \tan \theta_{s}-r_{m}\left(1-\cos \theta_{s}\right)}{\sin \theta_{s}}-l_{m}-C_{H} .
$$

5.4. Position Detection of Highest Step in Descend-Ready Phase. It is desirable for the robot to accurately perceive the relative position with the stairs in order to smoothly and safely perform the operation of the climbing down after the pitch-up descend phase. However, the information about the shape of the stairs that can be perceived by the robot on the upper floor applies only to the top edge of the step. Therefore, in order to detect this using a robot that has no external sensors, we let the robot perform the following operation. First, bring the rear subtrack into contact with the ground, and tilt the robot body forward slightly. More specifically, the 


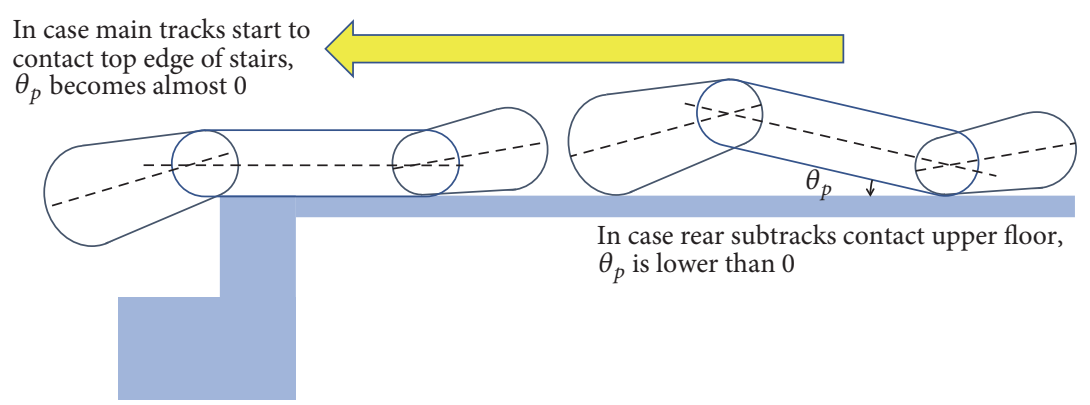

FIgURE 12: Position detection when the robot starts climbing down.

pitch angle of the robot body is set to an angle that exceeds the detection error range of the pitch angle $e_{p}$, and the stability margin [17] is sufficient. We define this angle as $\theta_{\mathrm{de}}$. On the other hand, with respect to the front subtrack, the position is determined so that it stays very close to the upper horizontal floor such that the forward tilting can be performed smoothly and safely. In summary, the target joint angle of the subtracks can be expressed as the following equations:

$$
\begin{aligned}
& \theta_{f}^{\mathrm{ref}}=-\theta_{p}+\Delta_{p}+\delta, \\
& \theta_{r}^{\mathrm{ref}}=\theta_{\mathrm{de}} .
\end{aligned}
$$

As described above, if the robot moves backward to the stair while controlling the subtracks, the robot can perceive that it is in the relative position shown in Figure 12 when the pitch angle of the robot body becomes horizontal.

5.5. Shock Mitigation in Pitch-Up Descend Phase. In the pitchup descend phase, it is very important to mitigate the shock when the body collapses to the stairs immediately after the centroid of the robot passes through the top edge of the stairs. In order to decrease this shock, $\theta_{p}$ should be made equal to $\theta_{s}$ as much as possible before the position of the centroid exceeds the edge of the highest step. Based on this idea, the target joint angle of each subtrack is determined as follows. First, the front subtrack is controlled so that the inclination of the robot body $\theta_{p}$ matches the inclination of the stairs $\theta_{s}$ as much as possible. Specifically, the feedback control described in the following equation is performed:

$$
\theta_{f}^{\mathrm{ref}}=K_{P}\left(\theta_{s}-\theta_{p}\right)+K_{I} \int\left(\theta_{s}-\theta_{p}\right) d t
$$

Equation (18) implies PI control of the front subtrack so that the pitch angle of the robot body $\theta_{p}$ matches the stair inclination $\theta_{s}$. However, based on this control method, it is necessary for it to be limited in $\theta_{f}^{\text {ref }} \leq \pi / 2+\theta_{p}$ as a countermeasure for the case where the length of the front subtrack is insufficient to make the robot body inclination equal to the inclination of the stairs (see Figure 13).

On the other hand, with respect to the rear subtrack, it needs to be controlled based on the following equation so that the inclination of the robot body coincides with the inclination of the stairs after the centroid position crosses the edge of the highest step:

$$
\theta_{r}^{\mathrm{ref}}=\theta_{s}-\theta_{p}+\Delta_{r}
$$

Further, switching from the immediately preceding descend-ready phase to the pitch-up descend phase can be performed when the inclination angle of the robot body $\theta_{p}$ becomes sufficiently horizontal. Specifically, switching should be performed when the following equation (20) is satisfied using the detection error in the pitch angle direction $e_{p}$

$$
\theta_{p} \geq-e_{p}
$$

5.6. Prevention of Falling Backward and Sideward in Normal Climbing-Down Phase. It is better to perform the same control as the normal climbing-up phase. That is, in order to prevent falling backward, the robot should extend the front and rear subtracks horizontally, and to prevent direction error, the main crawler should be controlled so that the robot is directed perpendicular to the edges of the stairs based on the roll-angle information. It is reasonable that switching from the immediately preceding pitch-up descend phase is performed when it is believed that the position of the centroid of the robot has moved downward from the edge of the highest step, as well as when the pitch angle of the robot body $\theta_{p}$ matches the inclination of the stairs $\theta_{s}$. Here, we describe the joint angle of the front subtrack in this situation. Based on Figure 14, the following equation holds when the $\mathrm{ZMP}$ is positioned at the vertex of the uppermost stage of the staircase:

$$
\begin{gathered}
x-C_{L} \cos \theta_{s}-\left(C_{H}-r_{m}\right) \sin \theta_{s}=0, \\
\therefore x=C_{L} \cos \theta_{s}+\left(C_{H}-r_{m}\right) \sin \theta_{s},
\end{gathered}
$$

where $x$ is the horizontal distance between the center of the sprocket in front of the main tracks and the top of the staircase when ZMP is coincident with the top edge of the stair. In addition, let $y$ be the distance in the vertical direction between the sprocket center and the upper floor in front of the main track in the same state, and when $\theta_{p}$ matches $\theta_{s}$, it is described as

$$
y=x \tan \theta_{s}+\frac{r_{m}}{\cos \theta_{s}}
$$


Robot can match $\theta_{p}$ to $\theta_{s}$ with front subtracks

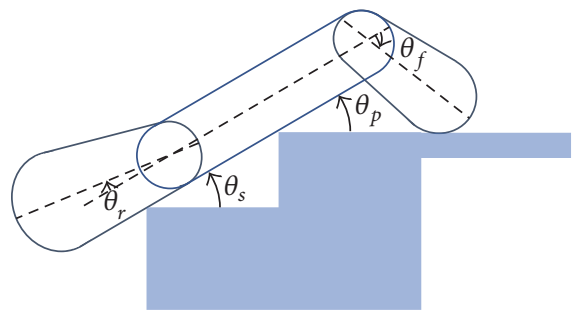

(a) $\theta_{s}$ is small
Robot can not match $\theta_{p}$ to $\theta_{s}$ with front substracks In case $\theta_{f}=-\theta_{s}-\pi / 2, \theta_{p}$ maximizes

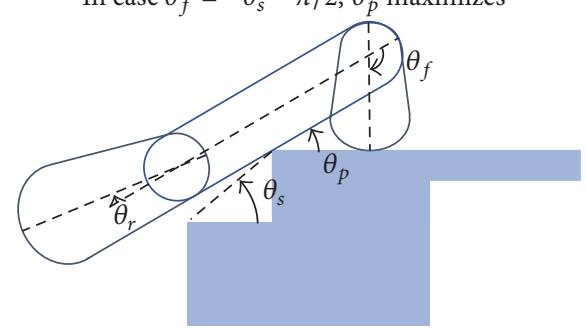

(b) $\theta_{s}$ is large

FIgURE 13: Pitch-up descend.

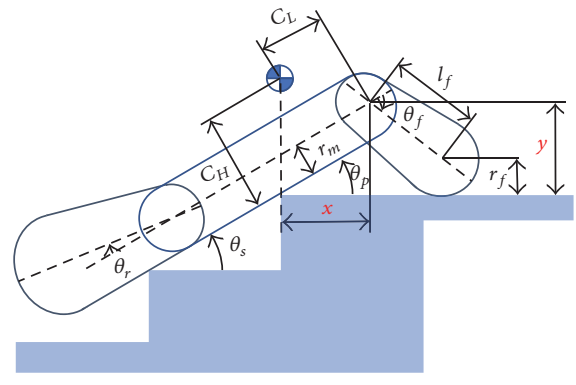

FIGURE 14: State to switch to normal climbing-down phase.

Moreover,

$$
y=r+l_{f} \sin \left(\theta_{f}-\theta_{s}\right) .
$$

Therefore, we can derive the following equation:

$$
\theta_{f}=-\theta_{s}+\arcsin \left(\frac{r_{f}-x \tan \theta_{s}-r_{m} / \cos \theta_{s}}{l_{f}}\right) \text {. }
$$

From the above, it is reasonable to switch when both (25) and (26) are satisfied.

$$
\begin{aligned}
& \theta_{f}>-\theta_{s}+\arcsin \left(\frac{r_{f}-x \tan \theta_{s}-r_{m} / \cos \theta_{s}}{l_{f}}\right), \\
& \theta_{p}>\theta_{s}-e_{p} .
\end{aligned}
$$

Here, when $l_{f}<\left|r_{f}-x \tan \theta_{s}-r_{m} / \cos \theta_{s}\right|$, the arcsin term in (25) cannot be defined. This means that when the pitch angle of the robot body coincides with the staircase, and the position of the centroid exceeds the highest edge of the stairs, the front subcrawler is too short to touch the upper floor. In this case, it is reasonable to ignore the condition of (25).

\subsection{Preventing Sliding Down and Impact Mitigation in the} Pitch-Down Descend Phase. In order to avoid the shock shown in Figure 7, it is necessary to set the front subtrack to the upper direction than the step inclination and to set the fall height to 0 before leaving. Alternately, if the length of the front subtrack is insufficient to touch the upper floor, it is desirable to make it as small as possible. In addition, it is necessary to consider shocks that can occur due to slipping. Here, as described in Section 5.1, the joint angle of the front subtrack that most prevents slipping is expressed by (3), which means that it is always directed upward than the inclination of the robot body in most cases. In this study, we focus on the prevention of slippage, and we assume that the falling height can be reduced to zero because the front subtrack is sufficiently long, or even if a drop of a certain height occurs, the impact will be allowable. According to this assumption, the operational concept of each subtrack is similar to that described in Section 5.1, and it is reasonable to perform control based on (3) and (4).

Next, we consider a reasonable condition for switching the control method from the last normal climbing-down phase. It is an option to switch when the pitch angle of the robot body becomes smaller than the inclination angle of the stairs. However, switching the control method at this instant increases the pitch angle of the robot after the grounding point to the lower floor moves from the rear subtrack to the main track and generates the risk of falling backward. In this study, we assumed that the backward-falling motion does not occur when the inclination of the robot body is less than the inclination of the stairs. For this reason, it is safe to switch the control method after ensuring that the inclination of the robot body does not exceed the step inclination upon changing the control method. The state before and after switching at this moment is shown in Figure 15. Based on geometric conditions,

$$
\begin{aligned}
\sin \theta_{s} & =\frac{p \sin \theta_{s}-r_{m}\left(1-\cos \theta_{s}\right)}{z} \\
\therefore z & =p-\frac{\left(1-\cos \theta_{s}\right)}{\sin \theta_{s}} r_{m}
\end{aligned}
$$

is established. Moreover, we also derived the following equation:

$$
\begin{aligned}
\sin \theta_{p}= & \frac{p \sin \theta_{s}-r_{r}\left(1-\cos \theta_{p}\right)}{l_{r} \cos \Delta_{r}+z} \\
\therefore \theta_{p}= & \arcsin \left(\frac{p \sin \theta_{s}-r_{r}}{\sqrt{\left(l_{r} \cos \Delta_{r}+z\right)^{2}+r_{r}^{2}}}\right) \\
& +\arctan \left(\frac{r_{r}}{l_{r} \cos \Delta_{r}+z}\right) .
\end{aligned}
$$



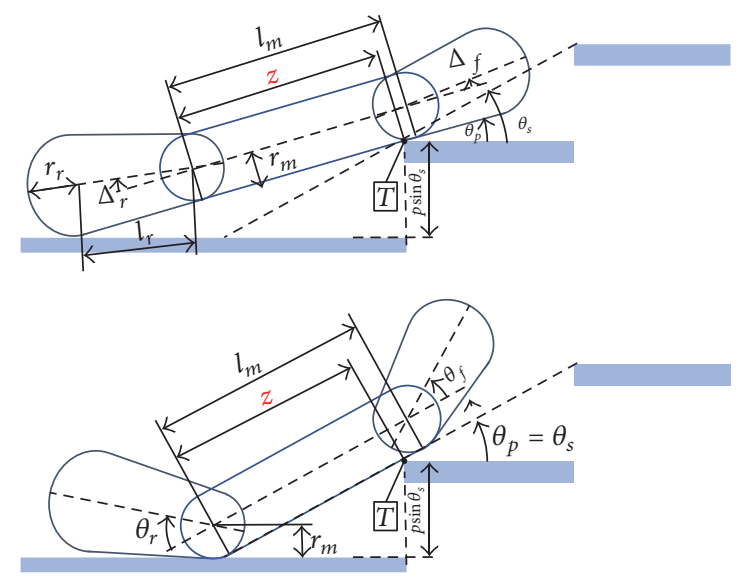

FIGURE 15: State before and after switching the control method.

From the above, it is considered safe to switch when the pitch angle of the robot body $\theta_{s}$ satisfies the following equation:

$$
\begin{gathered}
\theta_{p}<\arcsin \left(\frac{p \sin \theta_{s}-r_{r}}{\sqrt{\left(l_{r} \cos \Delta_{r}+z\right)^{2}+r_{r}^{2}}}\right) \\
+\arctan \left(\frac{r_{r}}{l_{r} \cos \Delta_{r}+z}\right)-e_{p} .
\end{gathered}
$$

\section{Verification Test}

We verified our proposed method described in the previous section by conducting tests with a tracked robot on mockup stairs. In this section, we describe the procedures and results, and we discuss the verification tests.

\subsection{Test Equipment}

6.1.1. Tracked Robot. We used the tracked robot Kenaf [18] in our laboratory for verification tests (Figure 16). Kenaf is a 6-DOF tracked robot that has two main tracks for traversal, as well as four subtracks, which are located on both sides at the front and rear of the robot and which can be controlled independently. However, the left and right subtracks are synchronized in the verification tests. Table 1 indicates the parameters of Kenaf. In addition, this robot is equipped with an RT-USB-9AXIS-00, which is a 9-axis IMU sensor module manufactured by RT Co., Ltd., in the main track part, and uses the posture-estimation filter proposed by Madgwick et al. [19]. Therefore, the robot can detect the pitch and roll angle with respect to the gravity direction.

In addition, the robot has a 9-axis IMU sensor module RT-USB-9AXIS-00 made by RT Co., Ltd., on the main tracks to detect the pitch and roll angles of the robot with respect to the gravity direction.

6.1.2. Mockup Stairs. In order to confirm whether the proposed method described in Section 5 can be applied even when the inclination angle $\theta_{s}$ and the pitch $p$ of the stair

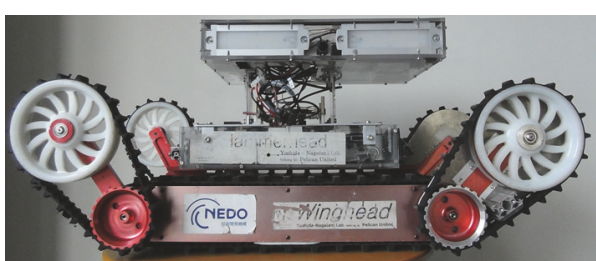

FIGURE 16: Tracked robot used in tests.

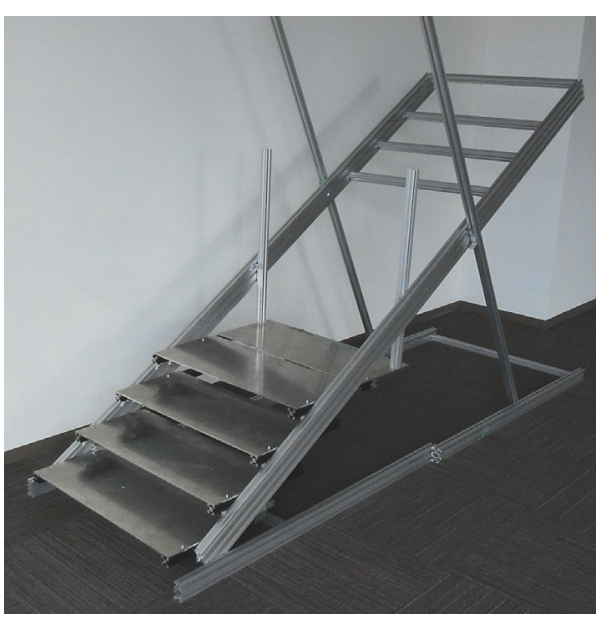

Figure 17: Changeable mockup stairs.

TABLE 1: Specification of the robot.

\begin{tabular}{lcc}
\hline Parameter & Symbol & Value \\
\hline Length of flat area in front ST & $l_{f}$ & $155[\mathrm{~mm}]$ \\
Length of flat area in MT & $l_{m}$ & $470[\mathrm{~mm}]$ \\
Length of flat area in rear ST & $l_{r}$ & $155[\mathrm{~mm}]$ \\
Radius of circular part in front ST & $r_{f}$ & $84[\mathrm{~mm}]$ \\
Radius of circular part in MT & $r_{m}$ & $56[\mathrm{~mm}]$ \\
Radius of circular part in rear ST & $r_{r}$ & $84[\mathrm{~mm}]$ \\
Mass of front ST & $m_{f}$ & $2.1[\mathrm{~kg}]$ \\
Mass of MT & $m_{m}$ & $22.8[\mathrm{~kg}]$ \\
Mass of rear ST & $m_{r}$ & $1.6[\mathrm{~kg}]$ \\
Centroid of $x$-coordinate of front ST & $x_{f}$ & $15[\mathrm{~mm}]$ \\
Centroid of $y$-coordinate of front ST & $y_{f}$ & $15[\mathrm{~mm}]$ \\
Centroid of $x$-coordinate of MT & $x_{m}$ & $0[\mathrm{~mm}]$ \\
Centroid of $y$-coordinate of MT & $y_{m}$ & $45[\mathrm{~mm}]$ \\
Centroid of $x$-coordinate of rear ST & $x_{r}$ & $-10[\mathrm{~mm}]$ \\
Centroid of $y$-coordinate of rear ST & $y_{r}$ & $16[\mathrm{~mm}]$ \\
\hline
\end{tabular}

MT: main tracks; ST: subtracks.

are changed, we used a self-made mockup stair, as shown in Figure 17. The stair can change both the pitch $p$ between two adjacent edges of the stairs and its inclination $\theta_{s} . \theta_{s}$ can be changed by up to $60^{\circ}$, and $p$ can be changed to any value up to $2400 \mathrm{~mm}$.

6.2. Test Procedure. We implemented the proposed method on Kenaf, and we conducted traversal tests on the mockup stairs with a safety rope in front of the robot. As the initial condition, the robot is made to face the step, and it started 
TABLE 2: Relationship between motion phases and failure modes.

\begin{tabular}{|c|c|c|c|c|c|}
\hline \multirow{2}{*}{\multicolumn{2}{|c|}{ Motion phases (MP) }} & \multicolumn{4}{|c|}{ Failure modes (FM) } \\
\hline & & I slipping & $\begin{array}{l}\text { II falling } \\
\text { backward }\end{array}$ & $\begin{array}{l}\text { III direction } \\
\text { error }\end{array}$ & IV excessive shock \\
\hline \multirow{3}{*}{ Ascend } & (i) Entering & Easy to occur and critical & \multicolumn{2}{|c|}{ Hard to occur } & $\begin{array}{l}\text { Does not happen if the } \\
\text { slipping is prevented }\end{array}$ \\
\hline & (ii) Normal climbing & $\begin{array}{l}\text { (i) Hard to occur } \\
\text { (ii) Not critical }\end{array}$ & \multicolumn{2}{|c|}{ Easy to occur and critical } & $\begin{array}{l}\text { (i) Hard to occur } \\
\text { (ii) Not critical }\end{array}$ \\
\hline & (iii) Leaving & $\begin{array}{l}\text { because of grousers } \\
\text { (=Assumption) }\end{array}$ & \multicolumn{2}{|c|}{$\begin{array}{c}\text { Hard to occur if it was } \\
\text { prevented in normal } \\
\text { climbing }\end{array}$} & Easy to occur and critical \\
\hline \multirow{4}{*}{ Descend } & (i) Ready & & Hard to occl & & \\
\hline & (ii) Entering & Hard to occur & $\begin{array}{l}\text { Easy to occu } \\
\text { and critical }\end{array}$ & Hard to occur & Easy to occur and critical \\
\hline & (iii) Normal climbing & $\begin{array}{l}\text { (i) Hard to occur } \\
\text { (ii) Not critical } \\
\text { because of grousers }\end{array}$ & \multicolumn{2}{|c|}{ Easy to occur and critical } & $\begin{array}{l}\text { (i) Hard to occur } \\
\text { (ii) Not critical }\end{array}$ \\
\hline & (iv) Leaving & $\begin{array}{l}\text { (i) Easy to occur } \\
\text { (ii) Not critical } \\
\text { except for excessive } \\
\text { shock }\end{array}$ & \multicolumn{2}{|c|}{$\begin{array}{l}\text { Hard to occur if it was } \\
\text { prevented in normal } \\
\text { climbing }\end{array}$} & Easy to occur and critical \\
\hline
\end{tabular}
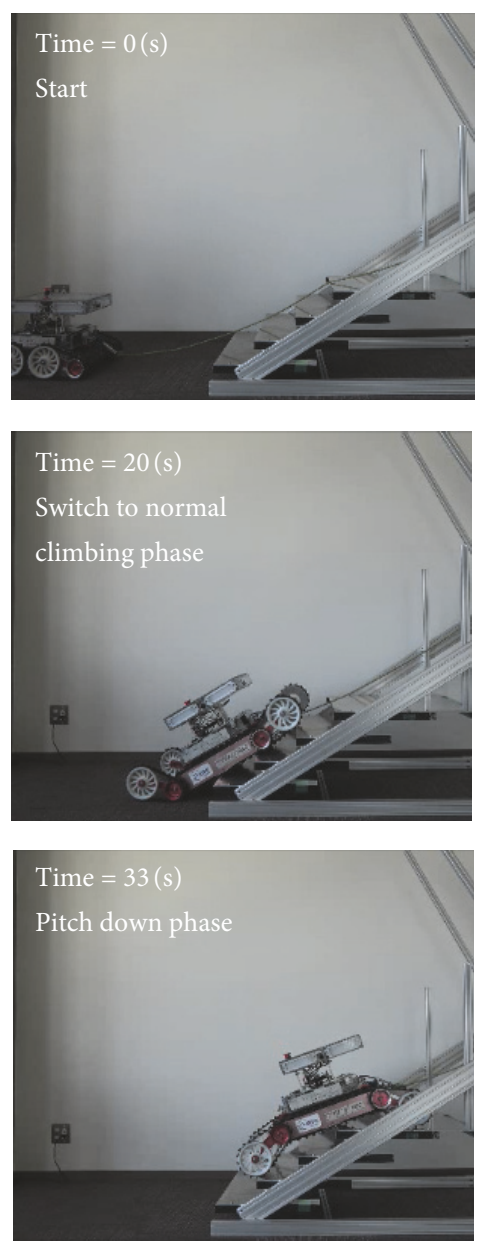
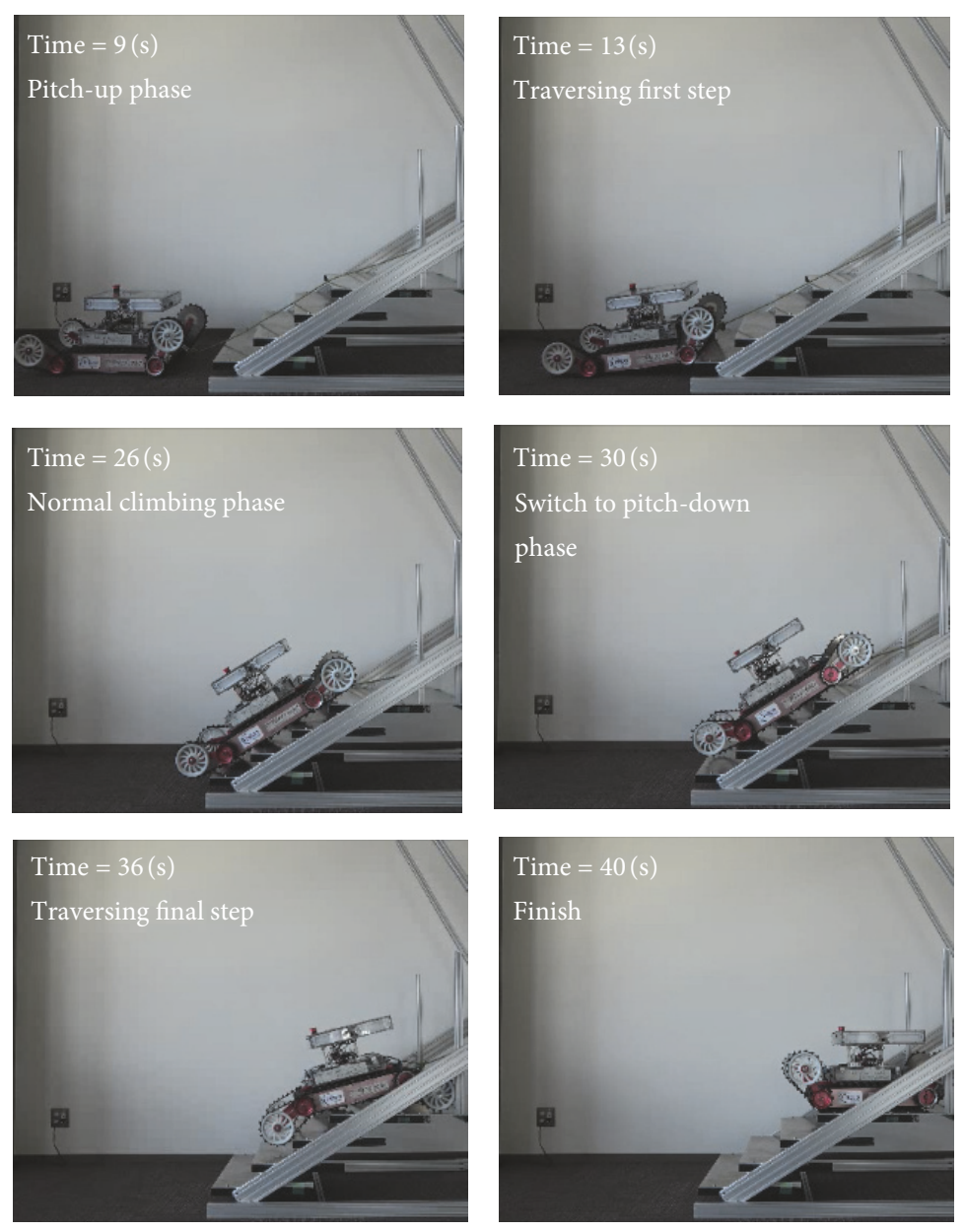

FIgURE 18: Climbing-up behavior for case $\theta_{s}=30^{\circ}, p=200 \mathrm{~mm}$. 

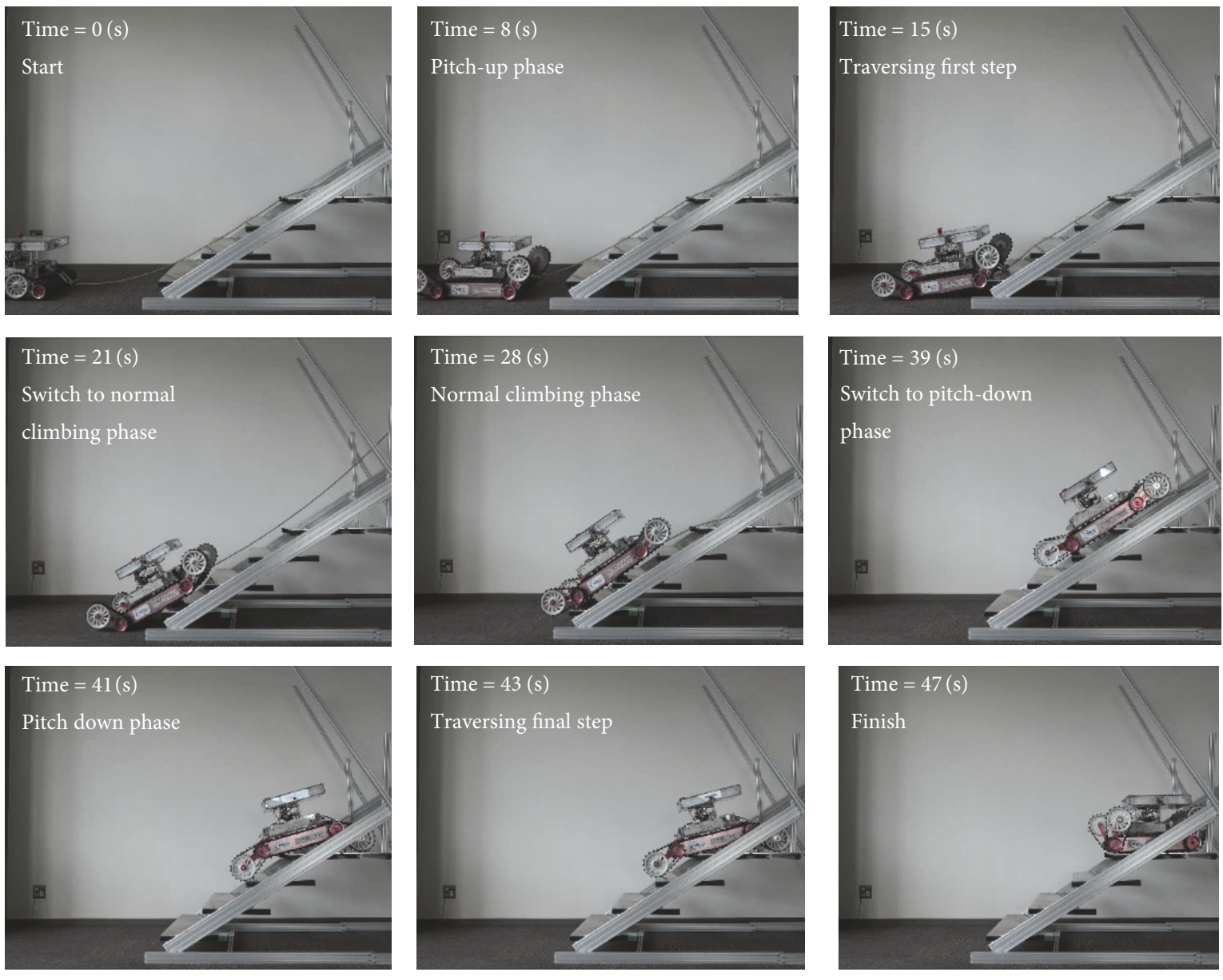

FIgURE 19: Climbing-up behavior for case $\theta_{s}=30^{\circ}, p=300 \mathrm{~mm}$.

with the front and rear subtracks folded inward $\left(\theta_{f}=\theta_{r}=\right.$ 165.2[ $\left.\left[^{\circ}\right]\right)$.

The running speed of the main tracks was set at $5 \mathrm{~cm} / \mathrm{s}$ and $-5 \mathrm{~cm} / \mathrm{s}$ for the ascending and descending tests, respectively. For (2) and (3), we set the friction coefficient to $\mu=$ 0.74 [-] based on our preliminary test. The margin of the rear subtrack required to prevent contact with the lower floor was set as $\delta=6^{\circ}$. The height of the free-fall margin $h$ was set at $50 \mathrm{~mm}$ for the condition $\theta_{s}=30^{\circ}$ and $80 \mathrm{~mm}$ for $\theta_{s}=45^{\circ}$. In addition, in the descend-ready phase, the target joint angle of the backward crawler $\theta_{\text {de }}$ was set to $-36^{\circ}$. According to the results of some preliminary experiments, the detection error of the pitch angle of the robot body was $e_{p}=2.9^{\circ}$.

On the other hand, based on the above parameters, we conducted verification tests under three different conditions, $\left(\theta_{s}, p\right)=\left(30^{\circ}, 200 \mathrm{~mm}\right),\left(30^{\circ}, 300 \mathrm{~mm}\right)$, and $\left(45^{\circ}, 200 \mathrm{~mm}\right)$, and we performed five trials for each condition. The number of steps was constantly found to be $n=4$.

In these tests, the pitch angle $\theta_{p}$ and the roll angle $\theta_{R}$ of the robot body, the joint angles $\theta_{f}, \theta_{r}$, and the operation mode were recorded as log information.

6.3. Test Results. Kenaf traversed the mockup stairs in all conditions without any failure modes. As a representative result in each condition, continuous photographs of the behavior of the robot are shown in Figures 18-23, and the data profiles obtained during these experiments are shown in Figures 24-29.

Based on the continuous photographs in Figures 18-23, we can confirm that the robot can safely climb up and down the stairs based on the proposed motion strategy. Here, we consider the transition of the state of the robot for each mode from the data profile shown in Figures 24-26, which is the test result of climbing up. First, in the pitch-up ascend phase, the pitch angle of the robot remained almost horizontal until it touched the lowest step, so the front and rear subtracks also kept almost constant angles according to (3) and (4). After the front subtrack makes contact with the lowest step, the pitch angle of the robot gradually increases, and along with this, the joint angle of the front subtrack is decreased and the rear subtrack is increased accordingly; therefore, we can see that the pitch angle of the main body is made to smoothly coincide with the stair-inclination angle. After that, when the pitch angle of the main body roughly matches the inclination angle of the stairs, it switches to the normal climbing-up ascend phase, and each joint angle of the front and rear subtracks is kept constant based on (5). In this way, it is possible to climb up stairs while maintaining the pitch angle of the robot body 

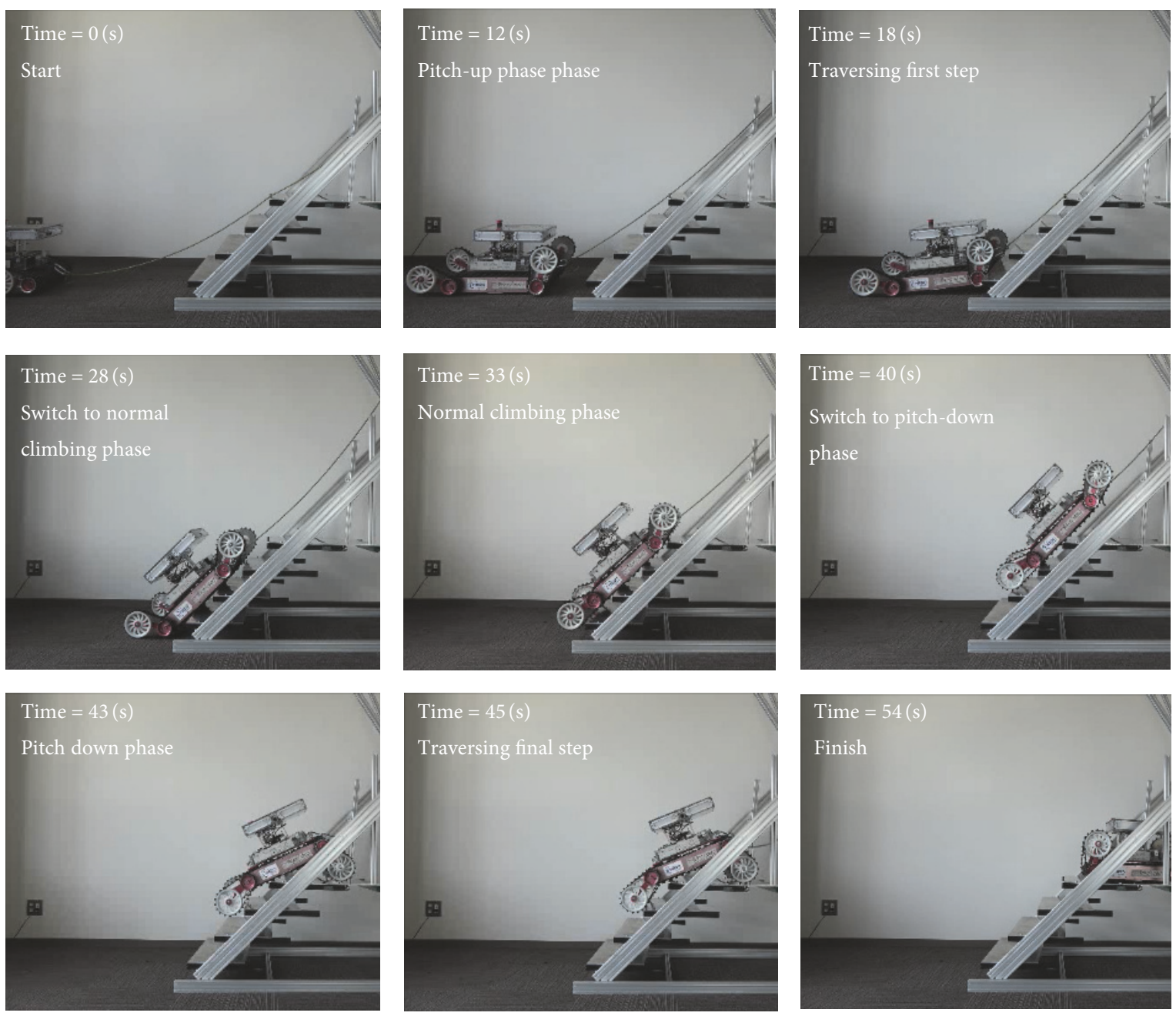

FIgURE 20: Climbing-up behavior for case $\theta_{s}=45^{\circ}, p=200 \mathrm{~mm}$.

almost at the inclination angle of the stairs. In this normal climbing-up phase, when the traveling distance satisfies (14), the transition to the pitch-down ascend phase is made, and the front and rear subtracks are determined based on (10) and (12). Then, it is possible to move the centroid on the upper floor and to decrease the pitch angle of the robot body.

On the other hand, we should consider the data profile shown in Figures 27-29, which is the test result of climbing down. In the first descend-ready phase, the robot went back keeping with its pitch angle of the robot body which was slightly decreased by controlling the joint angles of the front and rear subtracks based on (16) and (17). As a result, we see that condition (20) was established at approximately the same time that the rear end of the main track made contact with the highest edge of the stairs, and the transition to the subsequent pitch-up descend phase was made. In the pitch-up descend phase, the joint angles of the front and rear subtracks are controlled based on (18) and (19), respectively, and the pitch angle of the robot body can be made to coincide with the target inclination, $\theta_{s}$. After switching to the normal climbingdown phase, the robot continued to proceed in a backward direction until conditions (25) and (26) were established.
Then, it switched to the pitch-down descend phase. In this phase, the subtracks performed the same control as with the pitch-up ascend phase, and it successfully transitioned the pitch angle of the robot body horizontally.

Consequently, we applied the proposed method to various cases for different configurations of stairs.

\section{Conclusion}

In this paper, we described a method to make a 4-DOF tracked robot climb up and down a known flight of stairs autonomously based on internal sensors only. We classified both motion phases and four failure modes while the robot climbed up and down the stairs, and we then proposed an effective motion in each phase to prevent failure modes. Moreover, the transitions between phases were also realized based mainly on the inclination sensor of the robot body. As a result of the verification tests, we confirmed that a 4DOF tracked robot climbed up and down the stairs without any failure modes based on our proposed method. From the above, the objective of this research that is automatic floor change for the 4-DOF tracked robots through stairs was 

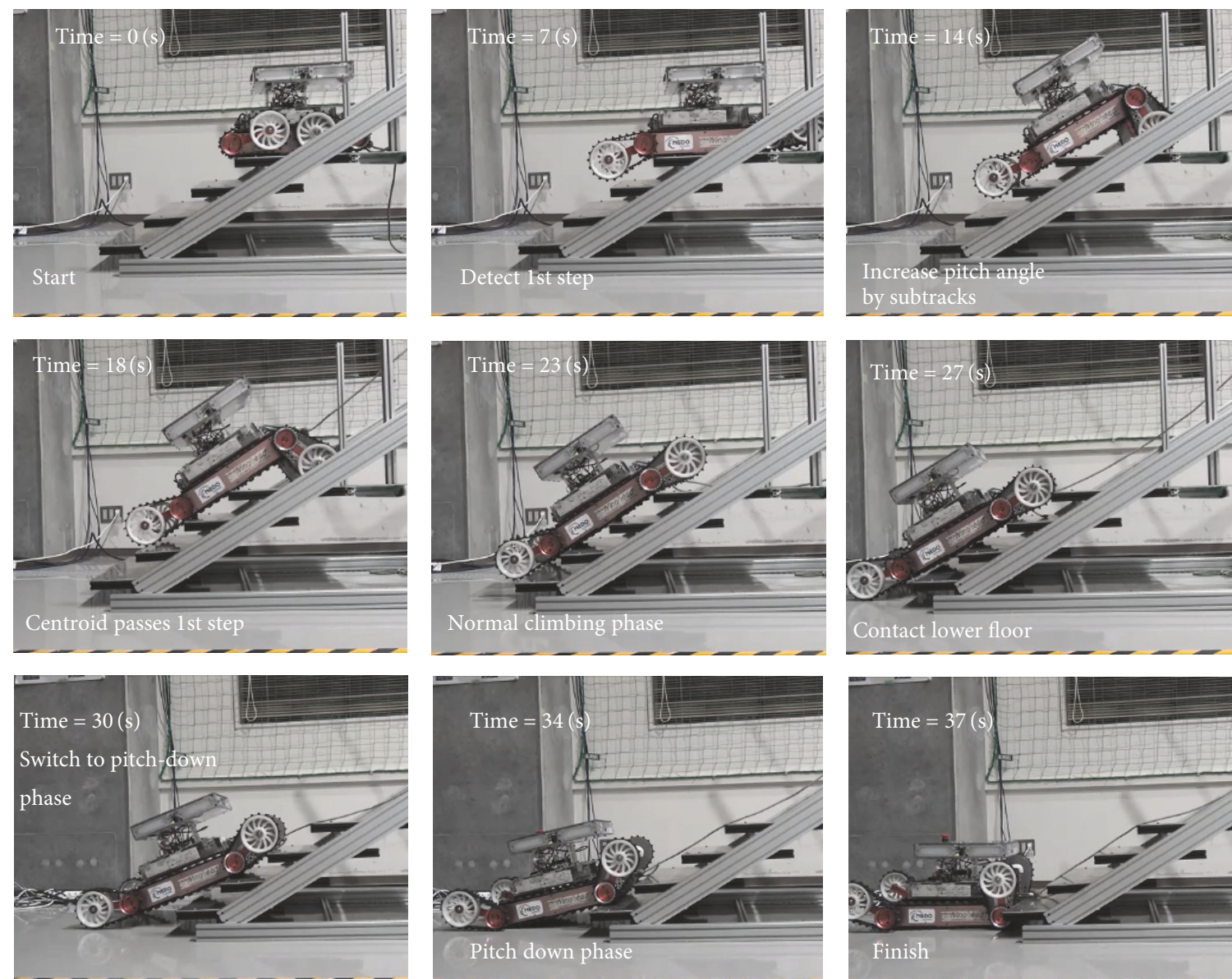

Figure 21: Climbing-down behavior for case $\theta_{s}=30^{\circ}, p=200 \mathrm{~mm}$.
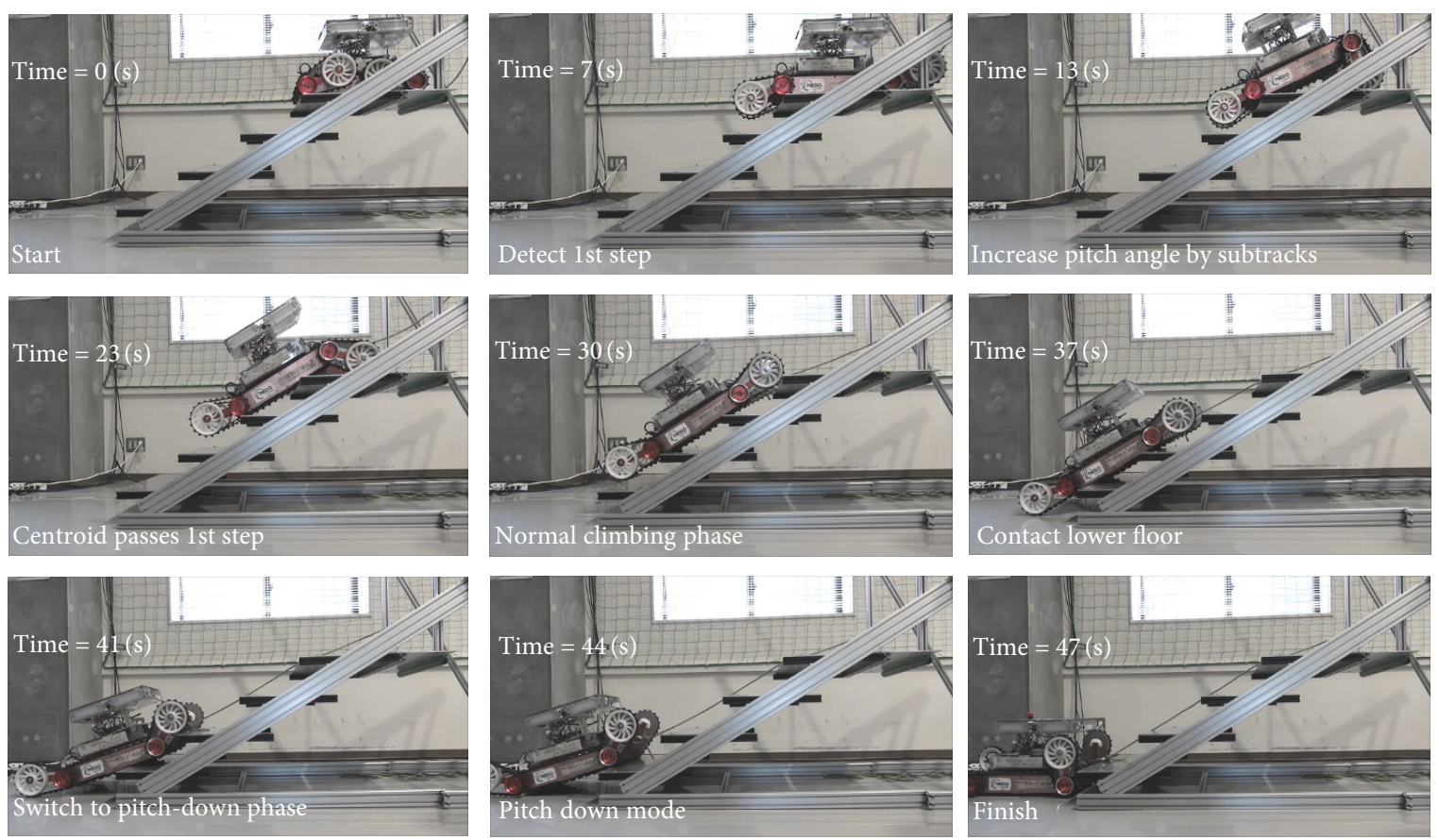

Figure 22: Climbing-down behavior for case $\theta_{s}=30^{\circ}, p=300 \mathrm{~mm}$. 

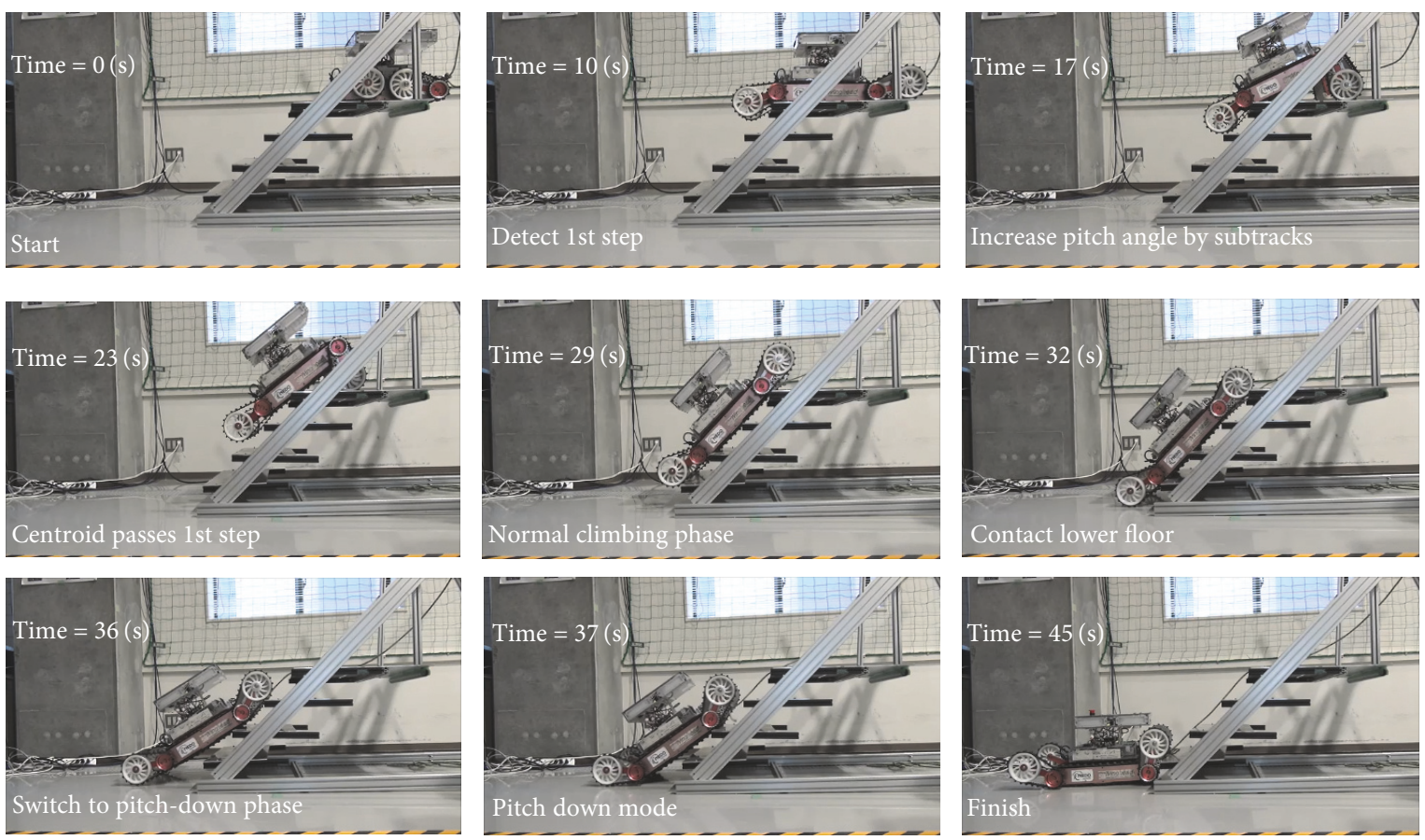

FIgURE 23: Climbing-down behavior for case $\theta_{s}=45^{\circ}, p=200 \mathrm{~mm}$.

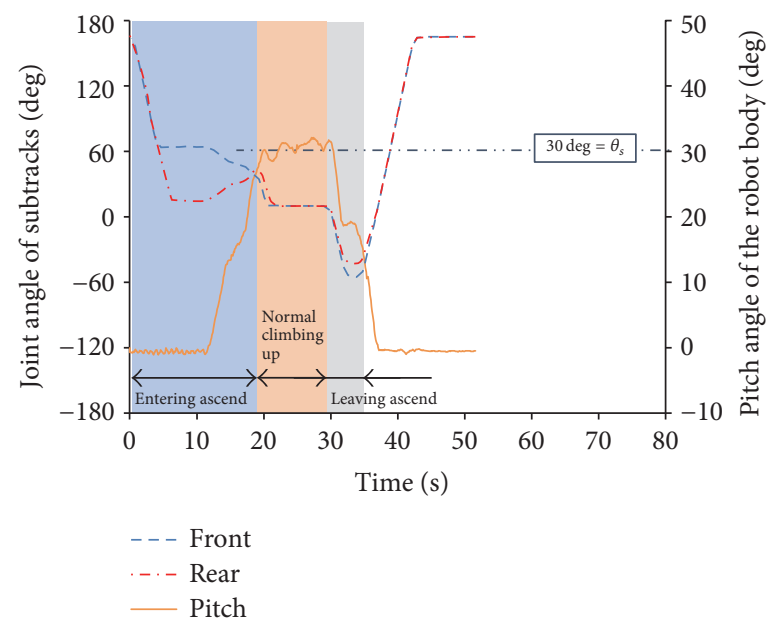

Figure 24: Data profile for $\theta_{f}, \theta_{r}, \theta_{p}\left(\theta_{s}=30^{\circ}, p=200 \mathrm{~mm}\right.$, ascend $)$.

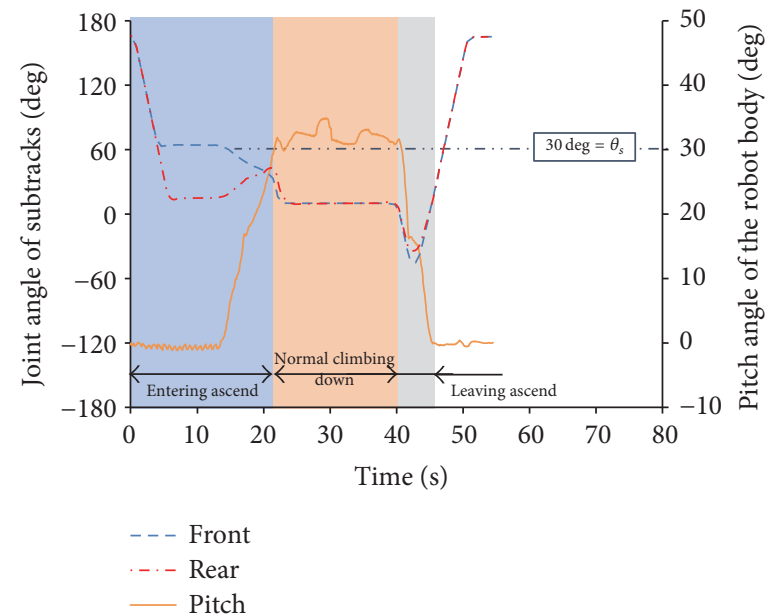

Figure 25: Data profile for $\theta_{f}, \theta_{r}, \theta_{p}\left(\theta_{s}=30^{\circ}, p=300 \mathrm{~mm}\right.$, ascend $)$. 


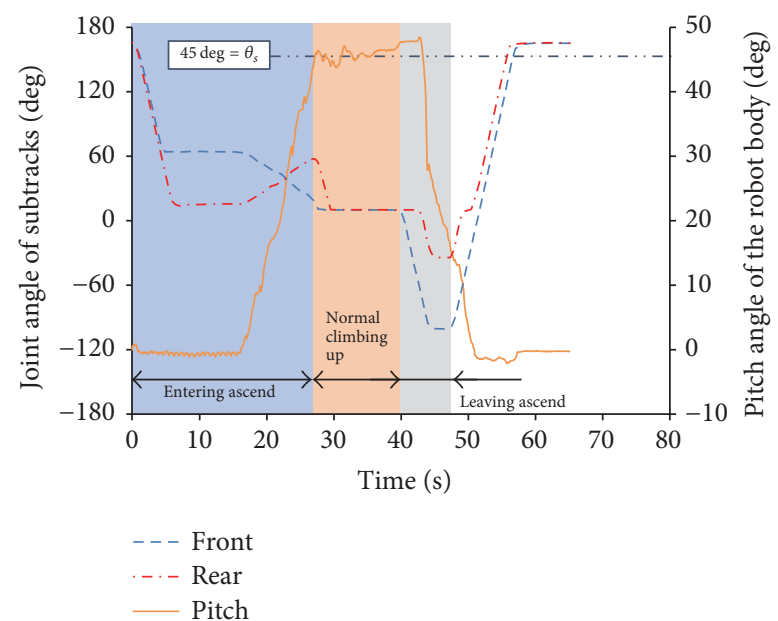

Figure 26: Data profile for $\theta_{f}, \theta_{r}, \theta_{p}\left(\theta_{s}=45^{\circ}, p=200 \mathrm{~mm}\right.$, ascend $)$.

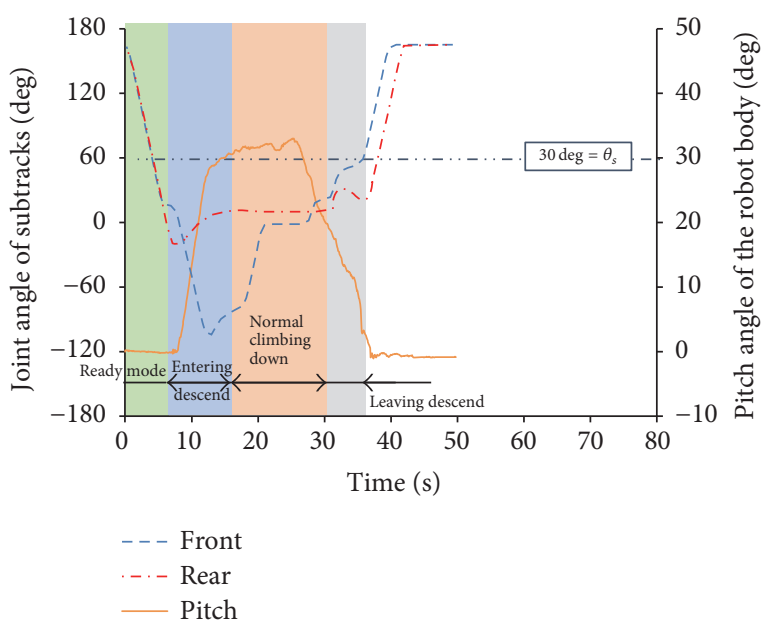

FIGURE 27: Data profile for $\theta_{f}, \theta_{r}, \theta_{p}\left(\theta_{s}=30^{\circ}, p=200 \mathrm{~mm}\right.$, descend).

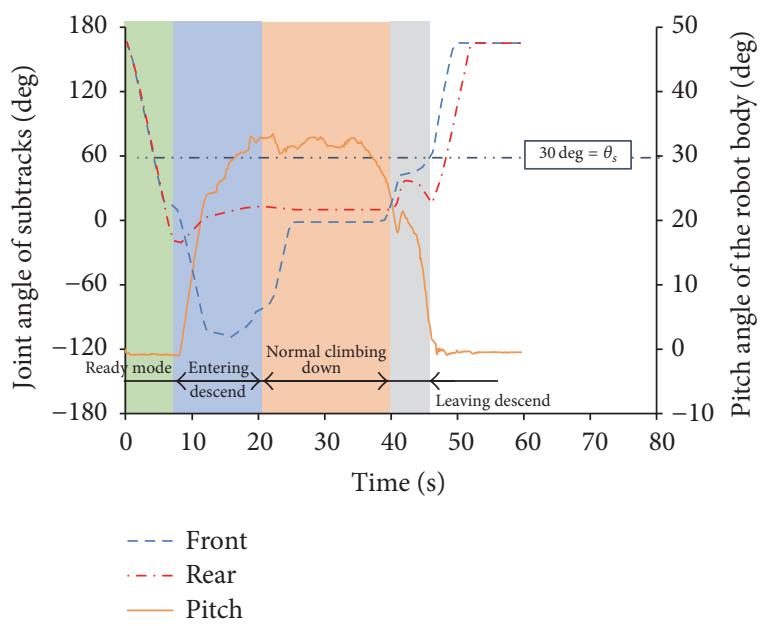

Figure 28: Data profile for $\theta_{f}, \theta_{r}, \theta_{p}\left(\theta_{s}=30^{\circ}, p=300 \mathrm{~mm}\right.$, descend $)$.

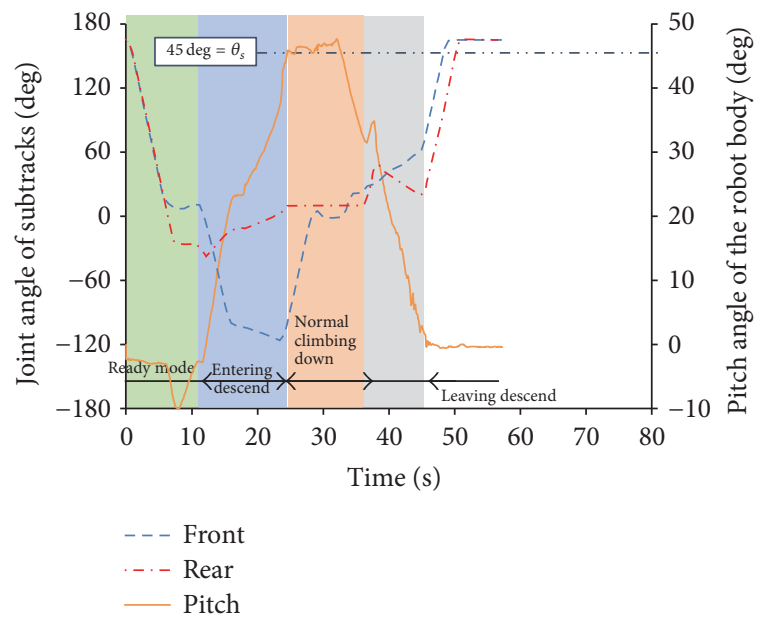

FIgURe 29: Data profile for $\theta_{f}, \theta_{r}, \theta_{p}\left(\theta_{s}=45^{\circ}, p=200 \mathrm{~mm}\right.$, descend $)$.

achieved. Moreover, the method described in this paper can be applied to many robots sharing the assumptions described in Section 2, and it can be expected to be widely generalized.

\section{Conflicts of Interest}

The authors declare that they have no conflicts of interest.

\section{Acknowledgments}

This work was supported in part by a Grant-in-Aid for JSPS Fellows (no. 16J2554). Finally, the authors would like to thank Editage (https://www.editage.jp) for the English language editing services that were provided.

\section{References}

[1] T. Yoshida, K. Nagatani, S. Tadokoro, T. Nishimura, and E. Koyanagi, "Improvements to the rescue robot quince toward future indoor surveillance missions in the Fukushima Daiichi nuclear power plant," in Proceedings of the 8th International Conference on Field and Service Robotics, 2012.

[2] K. Nagatani, S. Kiribayashi, Y. Okada et al., "Redesign of rescue mobile robot Quince," in Proceedings of the 9th IEEE International Symposium on Safety, Security, and Rescue Robotics (SSRR '11), pp. 13-18, Kyoto, Japan, November 2011.

[3] Y. Okada, K. Nagatani, K. Yoshida, T. Yoshida, and E. Koyanagi, "Shared autonomy system for tracked vehicles to traverse rough terrain based on continuous three-dimensional terrain scanning," in Proceedings of the 23rd IEEE/RSJ International Conference on Intelligent Robots and Systems (IROS '10), pp. 357362, Taipei, Taiwan, October 2010.

[4] A. I. Mourikis, N. Trawny, S. I. Roumeliotis, D. M. Helmick, and L. Matthies, "Autonomous stair climbing for tracked vehicles," International Journal of Robotics Research, vol. 26, no. 7, pp. 737758, 2007.

[5] I.-H. Li, W.-Y. Wang, and C.-K. Tseng, "A kinect-sensor-based tracked robot for exploring and climbing stairs," International Journal of Advanced Robotic Systems, vol. 11, no. 1, article A80, 2014. 
[6] A. Kalantari, E. Mihankhah, and S. A. A. Moosavian, "Safe autonomous stair climbing for a tracked mobile robot using a kinematics based controller," in Proceedings of the IEEE/ASME International Conference on Advanced Intelligent Mechatronics (AIM '09), pp. 1891-1896, Singapore, July 2009.

[7] E. Mihankhah, A. Kalantari, E. Aboosaeedan, H. D. Taghirad, and S. A. A. Moosavian, "Autonomous staircase detection and stair climbing for a tracked mobile robot using fuzzy controller," in Proceedings of the IEEE International Conference on Robotics and Biomimetics (ROBIO '08), pp. 1980-1985, IEEE, Bangkok, Thailand, February 2009.

[8] N. Li, S. Ma, B. Li, M. Wang, and Y. Wang, "An online stairclimbing control method for a transformable tracked robot," in Proceedings of the 2012 IEEE International Conference on Robotics and Automation, pp. 923-929, St Paul, Minn, USA, May 2012.

[9] Q. Zhang, S. S. Ge, and P. Y. Tao, "Autonomous stair climbing for mobile tracked robot," in Proceedings of the 9th IEEE International Symposium on Safety, Security, and Rescue Robotics (SSRR '11), pp. 92-98, Kyoto, Japan, November 2011.

[10] J. D. Martens and W. S. Newman, "Stabilization of a mobile robot climbing stairs," in Proceedings of the 1994 IEEE International Conference on Robotics and Automation, pp. 2501-2507, San Diego, Calif, USA, May 1994.

[11] P. Ben-Tzvi, S. Ito, and A. A. Goldenberg, "Autonomous stair climbing with reconfigurable tracked mobile robot," in Proceedings of the IEEE International Workshop on Robotic and Sensor Environments (ROSE '07), pp. 104-109, Ottawa, Canada, October 2007.

[12] K. Ohno, S. Morimura, S. Tadokoro, E. Koyanagi, and T. Yoshida, "Semi-autonomous control system of rescue crawler robot having flippers for getting over unknown-steps," in Proceedings of the IEEE/RSJ International Conference on Intelligent Robots and Systems (IROS '07), pp. 3012-3018, San Diego, Claif, USA, November 2007.

[13] D. Endo, A. Watanabe, and K. Nagatani, "Stair climbing control of 4-degrees-of-freedom tracked vehicle based on internal sensors," in Proceedings of the 14th International Symposium on Safety, Security and Rescue Robotics (SSRR '16), pp. 112-117, Lausanne, Switzerland, October 2016.

[14] Y. Liu and G. Liu, “Track-stair interaction analysis and online tipover prediction for a self-reconfigurable tracked mobile robot climbing stairs," IEEE/ASME Transactions on Mechatronics, vol. 14, no. 5, pp. 528-538, 2009.

[15] J. Liu, Y. Wang, S. Ma, and B. Li, "Analysis of stairs-climbing ability for a tracked reconfigurable modular robot," in Proceedings of the 2005 IEEE International Workshop on Safety, Security and Rescue Robotics, pp. 36-41, Kobe, Japan, June 2005.

[16] M. Vukobratovic, B. Branislav, and D. Surdilovic, "Zeromoment point-proper interpretation and new applications," in Proceedings of the IEEE-RAS International Conference on Humanoid Robots, pp. 237-244, 2001.

[17] Y. Kan, "Normalized energy stability margin: generalized stability criterion for walking vehicles," in Proceedings of CRAWLER '98, pp. 71-76, 1998.

[18] T. Yoshida, E. Koyanagi, S. Tadokoro et al., "A high mobility 6-crawler mobile robot "Kenaf"," in Proceedings of the 4th International Workshop on Synthetic Simulation and Robotics to Mitigate Earthquake Disaster (SRMED '07), p. 38, Atlanta, Ga, USA, July 2007.

[19] S. O. H. Madgwick, A. J. L. Harrison, and R. Vaidyanathan, "Estimation of IMU and MARG orientation using a gradient descent algorithm," in Proceedings of the IEEE International Conference on Rehabilitation Robotics (ICORR '11), pp. 1-7, Zurich, Switzerland, July 2011. 


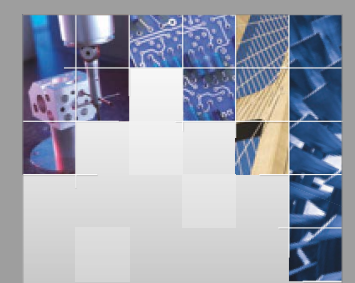

\section{Enfincering}
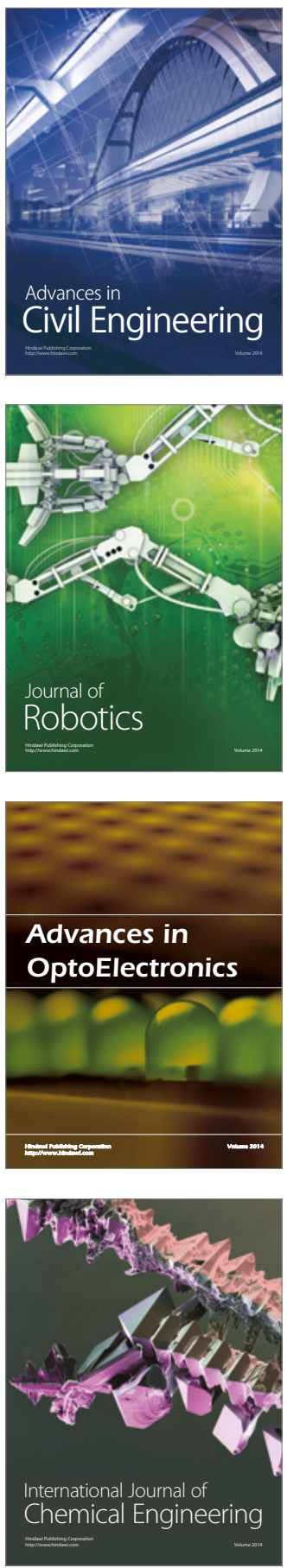

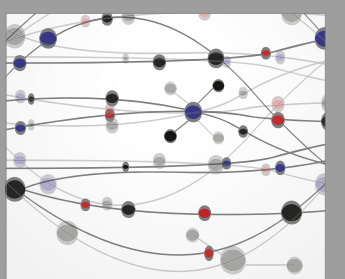

The Scientific World Journal

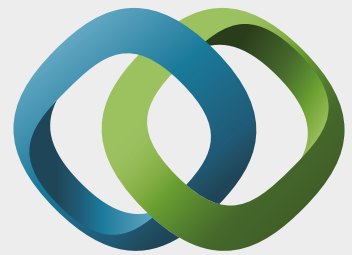

\section{Hindawi}

Submit your manuscripts at

https://www.hindawi.com
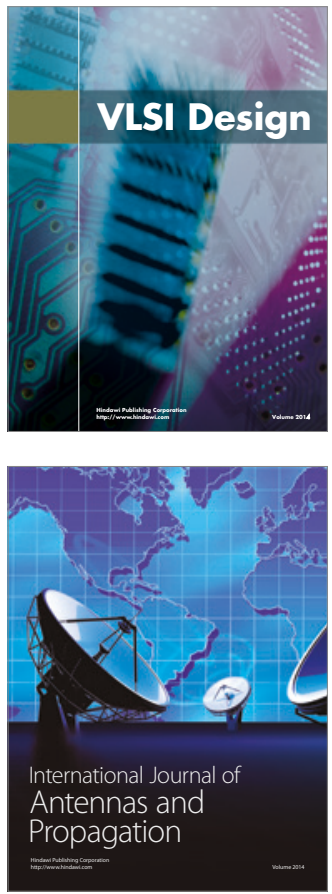

\section{Rotating}

Machinery
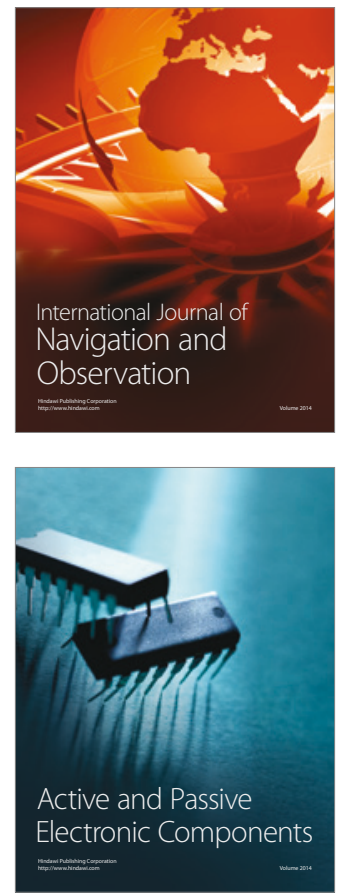
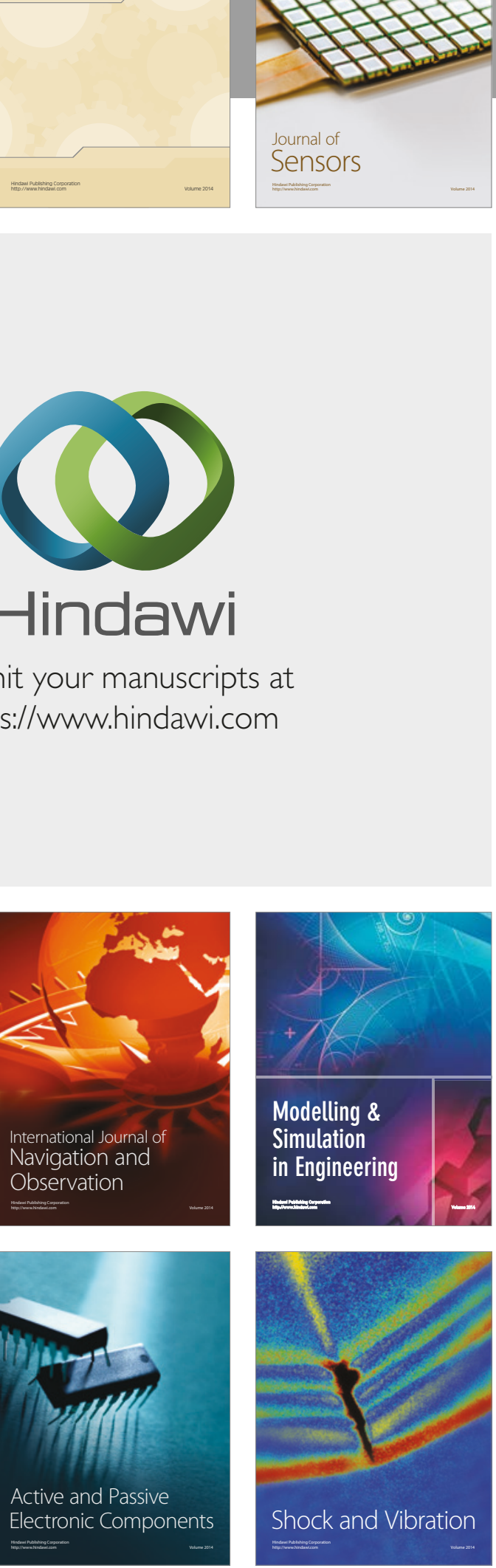
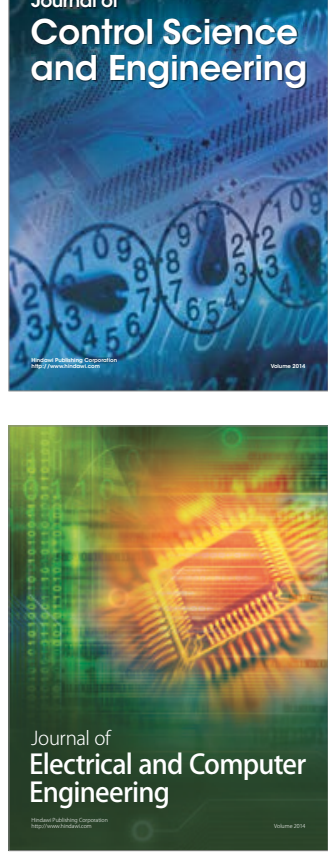

Distributed

Journal of

Control Science

and Engineering
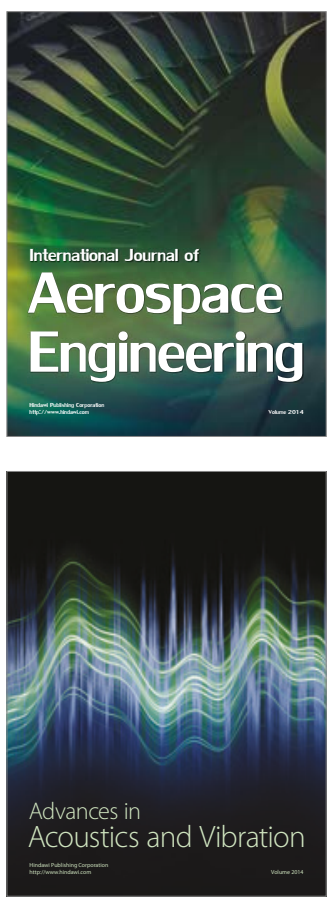

Sensor Networks 